March 14, 2017

PREPARED FOR SUBMISSION TO JCAP

\title{
Self-consistent Dark Matter Simplified Models with an s-channel scalar mediator
}

\author{
Nicole F. Bell, Giorgio Busoni and Isaac W. Sanderson \\ ARC Centre of Excellence for Particle Physics at the Terascale \\ School of Physics, The University of Melbourne, Victoria 3010, Australia \\ E-mail: n.bell@unimelb.edu.au, giorgio.busoni@unimelb.edu.au, \\ isanderson@student.unimelb.edu.au
}

\begin{abstract}
We examine Simplified Models in which fermionic DM interacts with Standard Model (SM) fermions via the exchange of an $s$-channel scalar mediator. The single-mediator version of this model is not gauge invariant, and instead we must consider models with two scalar mediators which mix and interfere. The minimal gauge invariant scenario involves the mixing of a new singlet scalar with the Standard Model Higgs boson, and is tightly constrained. We construct two Higgs doublet model (2HDM) extensions of this scenario, where the singlet mixes with the 2nd Higgs doublet. Compared with the one doublet model, this provides greater freedom for the masses and mixing angle of the scalar mediators, and their coupling to SM fermions. We outline constraints on these models, and discuss Yukawa structures that allow enhanced couplings, yet keep potentially dangerous flavour violating processes under control. We examine the direct detection phenomenology of these models, accounting for interference of the scalar mediators, and interference of different quarks in the nucleus. Regions of parameter space consistent with direct detection measurements are determined.
\end{abstract}




\section{Contents}

1 Introduction $\quad 1$

2 Restoring Gauge Invariance in S-channel Simplified Models 4

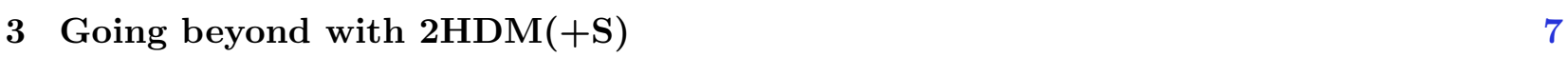

$3.12 \mathrm{HDM}+\mathrm{S}$ Scalar Spectrum 7

3.2 2HDM+S Lagrangian 9

$\begin{array}{lll}3.3 & \text { Yukawa structure } & 10\end{array}$

$\begin{array}{lll}\text { 3.3.1 } & \text { Type I,II,X and Y } & 10\end{array}$

3.3.2 Type III models and Minimal Flavour Violation 10

3.3.3 Aligned 2HDM+S 11

3.3.4 Coupling to only the first 2 generations $(2$ gen-2HDM+S) 12

4 Direct Detection $\quad 13$

4.1 Direct detection constraints for the $\mathrm{H}+\mathrm{S}$ model $\quad 14$

4.2 Direct detection constraints for $2 \mathrm{HDM}+\mathrm{S}$ scenarios 16

5 Conclusions $\quad 18$

A Unitarity, Stability and perturbativity of 2HDM+S $\quad 19$

$\begin{array}{ll}\text { A.1 Unitarity and perturbativity } & 19\end{array}$

B RGEs for 2-gen 2HDM+S 23

C Near-alignment and the $V_{\text {СКм }}$ pattern $\quad 25$

\section{Introduction}

The quest to uncover the identity of dark matter (DM) is one of the key challenges facing fundamental physics. Of the many types of dark matter candidates proposed, Weakly Interacting Massive Particles (WIMPs) are singled out as theoretically compelling candidates that can be meaningfully probed in current and forthcoming experiments. Indeed, the absence of a dark matter signal in the LHC run I data, together with complementary constraints from direct and indirect detection searches, has already excluded a non-trivial portion of the WIMP parameter space. The dark matter community is thus eagerly awaiting new data pertaining to the higher energy collisions of the LHC run II.

In order to interpret the results of these experiments, it is crucial to have a suitable framework to describe DM interactions with Standard Model (SM) particles. To this end, Effective Field Theories (EFTs) were used in many of the LHC $8 \mathrm{TeV}$ analyses. However, while an EFT approach is valid for low momentum transfer processes such in dark matter direct detection, it will break down at energy scales comparable to or larger than that of the new physics. Their use for LHC dark matter searches is thus not optimal, given the WIMP energy scale is expected to be comparable to the EW 
scale and hence directly accessible at the LHC. Indeed current LHC limits on the EFT energy scale fall in the range $\Lambda \sim O(\mathrm{GeV}-\mathrm{TeV})[1-6]$.

To remedy this issue, Simplified Models have been developed as a superior alternative that retain some of the desirable features of the EFTs (simple generic descriptions that approximate the relevant phenomenology of a broad spectrum of UV complete models) while remaining valid at higher energy scales. They do this through the explicit introduction of a particle that mediates the interactions of DM with SM fermions. The benchmark simplified models, outlined in the report of the LHC DM Forum (DMF), involve fermionic dark matter interacting via the exchange of a neutral spin-0 $s$-channel mediator, a neutral spin-1 $s$-channel mediator, or a charged spin- $0 t$-channel mediator [5-12].

While these simplified models are a definite improvement over the EFT approach, they are still not ideal. Indeed, some of the benchmark simplified models outlined by the DMF are clearly inadequate, as they are not gauge invariant and hence are not renormalizable. Issues associated with the lack of gauge invariance (with respect to both SM and dark-sector gauge groups) have been discussed in [13-19]. For example, models that involve the exchange of a spin-1 mediator $\left(Z^{\prime}\right)$ with axial-vector couplings to fermions is not gauge invariant unless a dark-Higgs is introduced to unitarize the longitudinal component of the $Z^{\prime}[17,18,20,21]$. The minimal self-consistent scenario involving an axial vector mediator must then be expanded to include two mediators - the $Z^{\prime}$ and scalar. The single mediator benchmark simplified models do not accurately capture the phenomenology of such two mediator models.

In this paper we consider the case of an $s$-channel scalar mediator. The most simplistic version of this scenario involves the introduction of a single new scalar, $S$, which mediates interactions between $\bar{\chi} \chi$ and $\bar{f} f$. However, because we assume the DM is not charged under the SM gauge symmetries, while $\bar{f} f=\bar{f}_{L} f_{R}+$ h.c. transforms as a $S U(2)_{L}$ doublet, this setup is not gauge invariant. This problem can be solved by mixing of the singlet scalar with the SM Higgs doublet, as in Refs.[22-30]. Like the $Z^{\prime}$ mediator case discussed above, we are thus forced to consider a two mediator model. However, as one of the two mixed scalar mediators is actually the SM Higgs boson, the parameter space of this model is quite constrained by measurement of the Higgs properties, and because all couplings to SM fermions are restricted to be proportional to SM Yukawa couplings.

This motivates a scenario in which the singlet scalar mixes not with the SM Higgs doublet, but with an additional doublet in a two Higgs doublet model (2HDM). The extension to 2HDM plus singlet has the advantage that we can approximately decouple the SM Higgs from the two mixed scalars that participate in DM interactions. Therefore, compared with the SM Higgs + singlet case:

- a greater range of scalar masses is possible, as neither of the two mixed scalars must have the $125 \mathrm{GeV}$ mass of the SM Higgs

- the couplings of the additional scalars to the SM fermions is not necessarily dictated by the SM Yukawa couplings.

In particular, we shall explore the scope for having different proportionality constants for the up-type quarks, down-type quarks and leptons, or flavour dependent couplings that are not proportional to the Standard Model Yukawa couplings.

Note that 2HDMs are very well motivated, arising in SUSY, GUTs and other extensions of the SM, and have been extensively studied [31-34]. The inclusion of an extra singlet scalar, 2HDM+S, 
occurs in the NMSSM version of SUSY [35], where in that application the singlet scalar is added to solve the $\mu$ problem [36, 37]. While the $2 \mathrm{HDM}+\mathrm{S}$ scenario [38-40] will allow a broader range of phenomenological possibilities for our dark matter application, it will also be subject to constraints. Chief among these, as with all 2HDMs, will be flavour changing bounds. We must therefore choose particular schemes in which potentially dangerous flavour changing couplings are kept under control, as will be discussed in detail below.

We shall explore the phenomenology of these mixed scalar mediator models to determine viable regions of parameter space that are consistent with current direct detection bounds. One feature of such models will be the interference of the amplitudes governed by the two mediators, which in some cases will lead to relative cancellation and hence suppression of cross sections. Such destructive interference is a generic feature of multi-mediator models, and has been discussed in Refs. [20, 24, 41], for the SM Higgs + singlet case. In our case, the effect is expanded to cover scalar masses that are unrelated to that of the SM Higgs. In addition, relative cancellations of contributions from different quarks will also suppress direct detection cross sections in some of the scenarios we consider.

The parameter space which survives the direct detection bounds will be of interest in the upcoming LHC run II analyses. Note that it is non-trivial to recast existing mono-X bounds to apply to the mixed scalar, due to the presence of the two interfering mediators (the exception, of course, is where one of the scalar mediators can be taken to decouple, effectively leaving a single mediator) and also due to the presence of additional signals, such as $\mathrm{W} / \mathrm{Z}+\mathrm{MET}$ and $\mathrm{VBF}+$ MET, which would contribute to the jets + MET signal. We defer a detailed collider analysis of the mixed scalar scenario to a future publication.

For scalar mediator models, indirect detection signals are expected to be too small to provide interesting constraints, as all the DM annihilation modes are p-wave suppressed by a factor of $v^{2} \sim 10^{-6}$ in the present universe (The p-wave suppression applies to both the $\bar{\chi} \chi \rightarrow \bar{f} f$ annihilation via a scalar mediator, and also the $\bar{\chi} \chi \rightarrow S S$ annihilation to a pair of scalars [42]). Note, though, that $\mathrm{p}$-wave processes may play a role at freezeout where the $v^{2}$ suppression factor is much less severe. Even so, it will be difficult to obtain the correct relic density in the SM Higgs + singlet model, except perhaps on resonance. The less restrictive $2 \mathrm{HDM}+\mathrm{S}$ model allows more freedom to reproduce the correct relic density, either via an s-channel resonance in $\bar{\chi} \chi \rightarrow \bar{f} f$ (i.e. $2 m_{\chi} \simeq m_{S}$, where $m_{S}$ is the mass of one of the mixed scalar states) or via annihilation into the scalars. Of course, adding additional particles into which the DM can efficiently annihilate, and which ultimately decay to the SM, is a way one can always fix the relic density, albeit at the expense of simplicity.

The case of a pseudoscalar mediator is very closely related to the scalar mediator models we consider. As with the scalar, the interaction of gauge singlet $\bar{\chi} \chi$ with an $\bar{f} \gamma_{5} f$ bilinear can only occur via the mixing of a SM-singlet pseudoscalar with an $S U(2)_{\mathrm{L}}$ doublet. However, given that the SM contains no pseudoscalar (after electroweak symmetry breaking), a mixed pseudoscalar plus 2HDM is the minimal scenario. Such models are discussed in detail in [43-48].

For the sake of clarity we shall use the following naming scheme to refer to the various scalar mediator models:

- Singlet scalar model (S). This is the non gauge invariant, single mediator, Simplified Model.

- Singlet scalar plus SM Higgs mixing model $(\mathrm{H}+\mathrm{S})$.

- Singlet scalar plus two Higgs doublet model (2HDM+S). 
The outline of the paper is as follows: We discuss the $\mathrm{S}$ and $\mathrm{H}+\mathrm{S}$ models and their constraints in Section 2, and outline possible $2 \mathrm{HDM}+\mathrm{S}$ extensions to these scenarios in Section 3. Direct detection bounds on all models are presented in Section 4.

\section{Restoring Gauge Invariance in S-channel Simplified Models}

The simplified models used by ATLAS and CMS for Dark Matter Searches (and included in recommendations for Run 2 [9]) are usually defined after electro-weak symmetry breaking. The standard framework for imposing MFV is to require that all couplings to quarks are proportional to the Standard Model Yukawas couplings, $y_{i}[5,7-9]$. The resulting Lagrangian is

$$
L=L_{\mathrm{SM}}+\frac{1}{2} \partial^{\mu} S \partial_{\mu} S-\frac{1}{2} M^{2} S^{2}-V_{\mathrm{int}}(S)-g_{q} S \sum_{q} \frac{y_{i}}{\sqrt{2}} \bar{q}_{i} q_{i}+\bar{\chi}\left(i \not \partial-\tilde{m}_{\chi}\right) \chi-y_{\chi} S \bar{\chi} \chi
$$

where $\chi$ is the DM, $q_{i}$ are the SM quarks, and $S$ is a singlet scalar mediator. The interaction part of the potential, $V_{\text {int }}(S)$, is usually neglected.

This Lagrangian is not invariant with respect to the $S U(2)_{L} \times U(1)_{Y}$ gauge symmetries. If $\chi$ is a $\mathrm{SM}$ singlet, the $S \bar{\chi} \chi$ vertex requires $S$ to also be a singlet. However, the SM bilinears $\bar{Q}_{L} u_{R}$ and $\bar{Q}_{L} d_{R}$ are not SM singlets, and can couple only to scalars that have the same quantum numbers as a Higgs doublet. Gauge invariance can be restored by allowing the singlet scalar $S$ to mix with either the SM Higgs $(\mathrm{H}+\mathrm{S}$ model), or with an additional Higgs doublet (2HMD+S model). We shall outline the $\mathrm{H}+\mathrm{S}$ model, and its constraints, and then turn to investigate whether it is possible to relax those constraints in the more general $2 \mathrm{HDM}+\mathrm{S}$ framework.

In the $\mathrm{H}+\mathrm{S}$ model, the scalar potential before EW symmetry breaking is given by

$$
V=-\frac{1}{2} M_{S S}^{2} S^{2}+\mu_{H S} \Phi^{\dagger} \Phi S+\frac{1}{2} \lambda_{H S} \Phi^{\dagger} \Phi S^{2}+\frac{1}{3 !} \mu_{S} S^{3}+\frac{1}{4 !} \lambda_{S} S^{4} .
$$

This potential is stable for $\lambda_{H S}>-\sqrt{\frac{2}{3} \lambda \lambda_{S}}$, where $\lambda$ is the SM Higgs quartic coupling. One may impose a $Z_{2}$ symmetry on the potential for $S$, thus discarding the $S^{3}$ and $\Phi^{\dagger} \Phi S$ terms. Although we shall make this choice for simplicity, we note that including these additional parameters could allow more freedom to enhance certain signals.

To permit the new scalar to couple to quarks, it needs to mix with the Higgs after EWSB, which requires that $\lambda_{H S},\langle\phi\rangle=v$ and $\langle S\rangle=w$ are nonzero. The condition $M_{S S}^{2}>\frac{1}{2} \lambda_{S H} v^{2}$ guarantees that $S$ acquires a vev. Defining the fields after symmetry breaking as

$$
\Phi=\left(\begin{array}{c}
G^{+} \\
\frac{v+h^{\prime}+G^{0}}{\sqrt{2}}
\end{array}\right) \text { and } S=w+s^{\prime}
$$

the mass matrix becomes

$$
M^{2}=\left(\begin{array}{cc}
2 \lambda v^{2} & \lambda_{S H} v w \\
\lambda_{S H} v w & \frac{1}{3} \lambda_{S} w^{2}
\end{array}\right) .
$$

This mass matrix can be diagonalised via a rotation to the mass eigenstate fields, $h=\cos \epsilon h^{\prime}+\sin \epsilon s^{\prime}$ and $s=-\sin \epsilon h^{\prime}+\cos \epsilon s^{\prime}$. As the Higgs Boson observed at the LHC is very SM-like, we take the 
mixing angle $\epsilon$ to be small by considering only small values for $\lambda_{S H}$. In this limit the mass eigenvalues are

$$
M_{h}^{2}=2 \lambda v^{2}+O\left(\lambda_{S H}^{2}\right) \text { and } M_{S}^{2}=\frac{1}{3} \lambda_{S} w^{2}+O\left(\lambda_{S H}^{2}\right)
$$

with mixing angle

$$
\tan \epsilon \simeq \frac{3 \lambda_{S H} v w}{6 \lambda v^{2}-\lambda_{S} w^{2}} \simeq \frac{\lambda_{S H} v w}{M_{h}^{2}-M_{S}^{2}} \simeq \sin \epsilon .
$$

The $h^{\prime}-s^{\prime}$ mixing allows both the $h$ and $s$ couple to Standard Model fermions and dark matter

$$
L_{\mathrm{int}, s-h}=-h \cos \epsilon \sum_{q} \frac{m_{i}}{v} \bar{q}_{i} q_{i}+s \sin \epsilon \sum_{q} \frac{m_{i}}{v} \bar{q}_{i} q_{i}-y_{D M}(s \cos \epsilon+h \sin \epsilon) \bar{\chi} \chi .
$$

The quark- $s$ couplings coincide with the ones of eq. ((2.1)) with

$$
g_{q} \equiv-\sin \epsilon .
$$

In this model, the proportionality constant $g_{q}$ is universal across generations. Indeed, the same proportionality constant applies not only for up and down quarks, but also for leptons. Importantly, both $s$ and $h$ mediate interactions between quarks and DM, so we have a two-mediator model.

When $m_{\chi}<M_{h} / 2$, the decay of the SM Higgs to DM gives a contribution to the Higgs invisible width of

$$
\Gamma_{h \rightarrow \chi \bar{\chi}}=\frac{y_{\chi}^{2} \sin ^{2} \epsilon}{8 \pi} M_{h}\left(1-\frac{4 m_{\chi}^{2}}{M_{H}^{2}}\right)^{3 / 2},
$$

which places tight constraints in this region of parameter space. ATLAS and CMS report upper limits of 0.78 [49] and 0.58 [50] at 95\% C.L. on the invisible branching fraction of the Higgs-boson. A combined analysis [51] also reports a lower bound on the Higgs signal strength $\mu$ of 0.87 . The quantity $\sin \epsilon$ is currently constrained by the Higgs signals strength to be $\sin \epsilon<0.4$ when $2 m_{\chi}<M_{h}$; for $2 m_{\chi}>M_{h}$ the Higgs invisible width provides no useful constraint. In addition to the interaction terms of eq. (2.7), there are other terms that are first order in $\sin \epsilon$, such as

$$
L_{\text {int }, s w z}=-\sin \epsilon\left(2 \frac{M_{W}^{2}}{v} W_{\mu}^{+} W^{-\mu}+\frac{M_{z}^{2}}{v} Z_{\mu} Z^{\mu}\right) s .
$$

All Standard model processes are only affected at second order in $\epsilon$ (apart from the ones involving Higgs cubic and quartic couplings). Nonetheless, this model is highly constrained by SM physics. For instance, precision electroweak constraints place upper limits on the mixing angle that range from $|\sin \epsilon|<0.4$ when $M_{S} \sim 200 \mathrm{GeV}$, to $|\sin \epsilon|<0.2$ when $M_{S} \sim 1000 \mathrm{GeV}$ [27].

Benchmark studies for this model can be found in [24,25], and relevant diagrams for monojet $+\mathbb{E}_{T}$ and $t \bar{t}+\mathbb{E}_{T}$ are shown in Fig. 1 . In addition, this model can lead to mono- $W / Z+\mathbb{E}_{T}, V B F+\mathbb{E}_{T}$ and even mono-Higgs $+\mathscr{E}_{T}$ processes, provided that we keep all the new terms arising from the scalar potential. Mono-Higgs processes depend on the trilinear vertices $h h S$ and $h S S$, and the relevant Feynman diagram is shown in Fig. 2.

The Monojet and $t \bar{t}+\mathbb{E}_{T}$ sensitivity for the $\mathrm{H}+\mathrm{S}$ model can be roughly estimated by rescaling that for the singlet $\mathrm{S}$ model, using

$$
\frac{\sigma_{S+H}}{\sigma_{S}} \sim \frac{\sin ^{2} \epsilon}{g_{q}^{2}} \lesssim \frac{0.4^{2}}{g_{q}^{2}}
$$




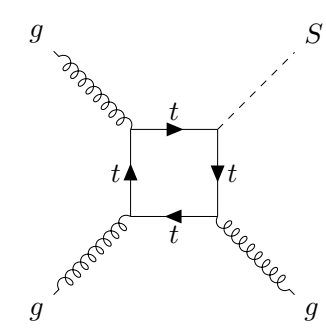

(a)

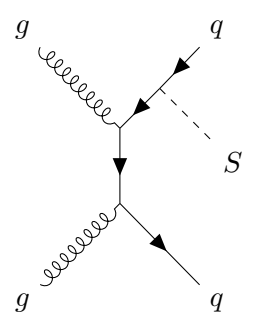

(e)

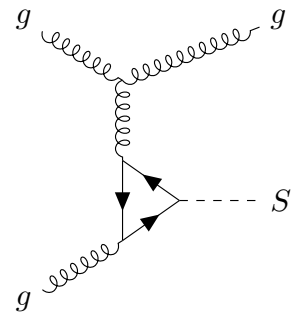

(b)

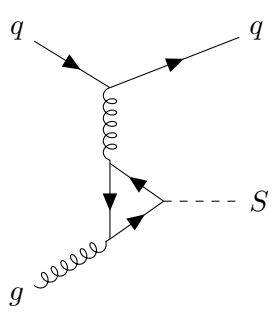

(c)

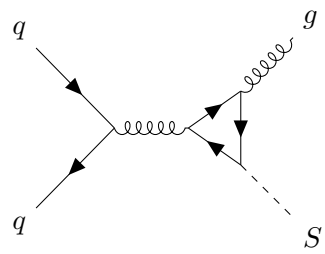

(d)

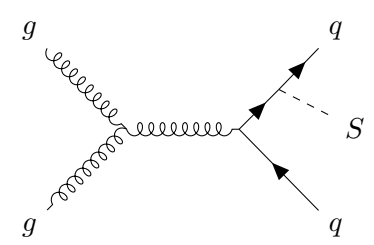

(f)

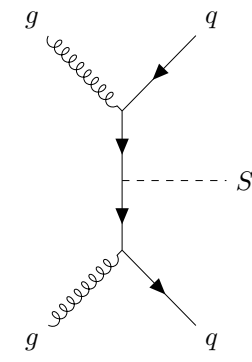

(g)

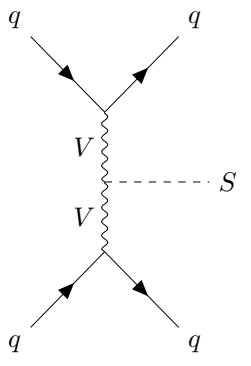

(h)

Figure 1: Feynman diagrams contributing to jets/mono-jet+ $\mathbb{E}_{T}$ signals in the $\mathrm{S}+\mathrm{H}$ model. Diagrams (e)-(h) also contribue to $t \bar{t}+\mathbb{E}_{T}$. All diagrams except (h) are also present in the S model.

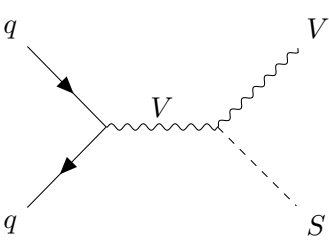

(a)

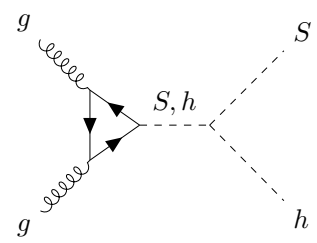

(b)

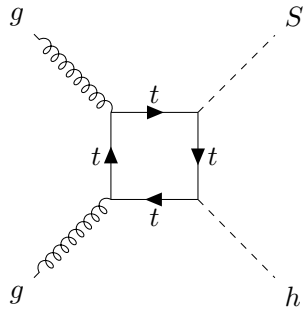

(c)

Figure 2: Feynman diagrams for mono- $W / Z+\mathbb{E}_{T}(\mathrm{a})$ and mono-Higgs (b and c).

Note that such a rescaling assumes the cross section are dominated by the new scalar mediator. If both the $s$ and $h$ mediators contribute, interference effects make such a rescaling invalid. Also note that this does not account for the contribution of the vector boson fusion operators of eq. (2.10) to the cross section in the $\mathrm{H}+\mathrm{S}$ model, so this approximation is valid as long as such operators are subdominant. The singlet $\mathrm{S}$ model has a rather small cross section, therefore the current exclusion limits are weak. CMS limits derived from $b \bar{b}+\mathbb{E}_{T}, t \bar{t}+\mathbb{E}_{T}$ and $j V+\mathbb{E}_{T}, V \rightarrow q \bar{q}$ final states are reported in [52-54] while ATLAS limits can be found in [55, 56]. CMS has nearly reached the sensitivity to exclude low mass mediators, for $g_{q}^{\text {sens }} \sim 1$. Using eq. (2.11), we estimate that the cross sections for the $\mathrm{H}+\mathrm{S}$ model will be smaller by a factor of $\sin ^{2} \epsilon /\left(g_{q}^{\text {sens }}\right)^{2} \lesssim 1 / 6.25$. This will make the model much harder to exclude, as it would require a luminosity roughly $6.25^{2} \sim 39$ times larger than for the singlet model, assuming a statistically limited scenario. 


\section{Going beyond with $2 \mathrm{HDM}(+\mathrm{S})$}

We now explain how to go beyond the standard set-up for section 2, in a scenario where gauge invariance is retained, but restrictions imposed by SM Higgs properties are relaxed or removed. In particular we shall explore the scope to:

- Have greater freedom for the couplings of the scalar mediator(s) to the SM fermions, including different proportionality constants for the coupling to the up quark, down quark and leptons sectors, or flavour-dependent couplings not forced to be proportional to SM Yukawa couplings.

- Have a range of scalar masses unconnected to the SM Higgs mass.

To achieve these aims, additional freedom with both the scalar mixing and Yukawa couplings is required. This can be achieved by adding a second Higgs doublet, thereby expanding the scalar sector to that of a $2 \mathrm{HDM}\left[31\right.$ ] plus a singlet scalar ${ }^{1}$. Below, we shall analyse the scalar spectrum of this scenario, and outline possible Yukawa structures that are consistent with flavour constraints. In doing so, we shall need to review and expand the pertinent features of $2 \mathrm{HDMs}$; Readers who are familiar with 2HDMs may wish to skip to the direct detection analysis in section 4 .

\subsection{HDM+S Scalar Spectrum}

The most general scalar potential we consider is

$$
V\left(\Phi_{1}, \Phi_{2}, S\right)=V_{2 \mathrm{HDM}}\left(\Phi_{1}, \Phi_{2}\right)+V_{S}(S)+V_{S 2 \mathrm{HDM}}\left(\Phi_{1}, \Phi_{2}, S\right)
$$

where $^{2}$

$$
\begin{aligned}
V_{2 \mathrm{HDM}}\left(\Phi_{1}, \Phi_{2}\right) & =M_{11}^{2} \Phi_{1}^{\dagger} \Phi_{1}+M_{22}^{2} \Phi_{2}^{\dagger} \Phi_{2}+\left(M_{12}^{2} \Phi_{2}^{\dagger} \Phi_{1}+h . c .\right)+\frac{\lambda_{1}}{2}\left(\Phi_{1}^{\dagger} \Phi_{1}\right)^{2}+\frac{\lambda_{2}}{2}\left(\Phi_{2}^{\dagger} \Phi_{2}\right)^{2} \\
& +\lambda_{3}\left(\Phi_{1}^{\dagger} \Phi_{1}\right)\left(\Phi_{2}^{\dagger} \Phi_{2}\right)+\lambda_{4}\left(\Phi_{2}^{\dagger} \Phi_{1}\right)\left(\Phi_{1}^{\dagger} \Phi_{2}\right) \\
& +\frac{1}{2}\left(\lambda_{5}\left(\Phi_{2}^{\dagger} \Phi_{1}\right)^{2}+\lambda_{6}\left(\Phi_{2}^{\dagger} \Phi_{1}\right)\left(\Phi_{1}^{\dagger} \Phi_{1}\right)+\lambda_{7}\left(\Phi_{2}^{\dagger} \Phi_{1}\right)\left(\Phi_{2}^{\dagger} \Phi_{2}\right)+\text { h.c. }\right) \\
V_{S}(S) & =\frac{1}{2} M_{S S}^{2} S^{2}+\frac{1}{3} \mu_{S} S^{3}+\frac{1}{4} \lambda_{S} S^{4} \\
V_{S 2 \mathrm{HDM}}\left(\Phi_{1}, \Phi_{2}, S\right) & =\mu_{11 S}\left(\Phi_{1}^{\dagger} \Phi_{1}\right) S+\mu_{22 S}\left(\Phi_{2}^{\dagger} \Phi_{2}\right) S+\left(\mu_{12 S} \Phi_{2}^{\dagger} \Phi_{1} S+\text { h.c. }\right) \\
& +\frac{\lambda_{11 S}}{2}\left(\Phi_{1}^{\dagger} \Phi_{1}\right) S^{2}+\frac{\lambda_{22 S}}{2}\left(\Phi_{2}^{\dagger} \Phi_{2}\right) S^{2}+\frac{1}{2}\left(\lambda_{12 S} \Phi_{2}^{\dagger} \Phi_{1} S^{2}+\text { h.c. }\right)
\end{aligned}
$$

To reduce the complexity of this model, we make several simplifying assumptions which are common with those made in many $2 \mathrm{HDM}$ studies. We assume CP is conserved in the Higgs sector, and is not spontaneously broken by a relative phase between the vevs of the 2 doublets. This implies that $M_{12}, \lambda_{5}, \mu_{12 S}, \lambda_{12 S}$ are real. We also impose a $Z_{2}$ symmetry on the potential $V_{2 \mathrm{HDM}}$, under which one of $\Phi_{1,2}$ is odd while the other is even, eliminating the $\lambda_{6,7}$ terms. However, we allow the $Z_{2}$ symmetry to be broken by soft terms, and thus keep the $M_{12}$ term. Moreover, as we are interested in

\footnotetext{
${ }^{1}$ Note that such a Higgs sector also arises in the NMSSM, where an additional singlet is added to solve the $\mu$ problem. In the NMSSM, however, the 2HDM couplings are forced to be of Type-II, while we will consider a broader and less restrictive range of Yukawa structures.

${ }^{2}$ Note that there are different conventions for the normalization of these coefficients; we have chosen the convention assumed in [31].
} 
building up a simplified model, we are primarily interested in the scalar mass spectrum and mixing, but not the scalar interactions. From this point of view, the parameters $\lambda_{i j s}$ and $\mu_{i j s}$ are equivalent ${ }^{3}$ so we choose to set the latter to zero. The same is true for $M_{S S}$ and $\mu_{S}$, so we discard the latter.

By writing the fields as

$$
\Phi_{i}=\left(\begin{array}{c}
\Phi_{i}^{+} \\
\frac{v_{i}+\rho_{i}+i \eta_{i}}{\sqrt{2}}
\end{array}\right), \quad S=v_{S}+\rho_{3},
$$

and using the mimima condition for the potential, we can eliminate the parameters $M_{11}, M_{12}, M_{S S}$ and replace them with $v_{1}, v_{2}, v_{S}$. The mass matrix for the scalars is built by a $2 \mathrm{x} 2$ block for the charged scalars (one of which will be the SM Goldstone boson $G^{+}$for the $W$ ), a $2 \times 2$ block for the pseudoscalars (one of which will be the SM Goldstone boson $G^{0}$ for the $Z$ ) and a $3 \times 3$ block for the 3 scalars. Defining the ratio of vevs in the usual way,

$$
\tan \beta=\frac{v_{2}}{v_{1}}, \quad \text { with } \quad v_{1}^{2}+v_{2}^{2}=v^{2},
$$

the non-zero eigenvalues for the physical charged scalar and pseudoscalar are

$$
\begin{aligned}
M_{H^{+}}^{2} & =\sec ^{2} \beta\left(M_{22}^{2}+\frac{1}{2} \lambda_{22 S} v_{S}^{2}\right)+\frac{v^{2}}{2}\left(\lambda_{2} \tan ^{2} \beta+\lambda_{3}\right), \\
M_{A}^{2} & =\sec ^{2} \beta\left(M_{22}^{2}+\frac{1}{2} \lambda_{22 S} v_{S}^{2}\right)+\frac{v^{2}}{2}\left(\lambda_{2} \tan ^{2} \beta+\lambda_{3}+\lambda_{4}-\lambda_{5}\right) .
\end{aligned}
$$

It is useful to perform a rotation to the so called "Higgs basis" where only one doublet obtains a vev

$$
\begin{gathered}
\Phi_{h}=\cos \beta \Phi_{1}+\sin \beta \Phi_{2}=\left(\begin{array}{c}
G^{+} \\
\frac{v+h+i G^{0}}{\sqrt{2}}
\end{array}\right), \\
\Phi_{H}=-\sin \beta \Phi_{1}+\cos \beta \Phi_{2}=\left(\begin{array}{c}
H^{+} \\
\frac{H+i A}{\sqrt{2}}
\end{array}\right) .
\end{gathered}
$$

The mass matrix for the scalars in the $\{h, H, S\}$ basis is

$$
M^{\rho}=\left(\begin{array}{ccc}
M_{h h}^{\rho} & M_{h H}^{\rho} & M_{h S}^{\rho} \\
M_{h H}^{\rho} & M_{H H}^{\rho} & M_{H S}^{\rho} \\
M_{h S}^{\rho} & M_{H S}^{\rho} & M_{S S}^{\rho}
\end{array}\right),
$$

where

$$
\begin{aligned}
M_{h h}^{\rho} & =\frac{\lambda_{1}+\lambda_{2}}{2} v^{2}+\frac{\lambda_{1}-\lambda_{2}}{2} v^{2} \cos 2 \beta-\frac{\lambda_{1}+\lambda_{2}-2 \lambda_{345}}{4} v^{2} \sin ^{2} 2 \beta, \\
M_{h H}^{\rho} & =-\frac{1}{4} v^{2} \sin 2 \beta\left(\lambda_{1}-\lambda_{2}+\left(\lambda_{1}+\lambda_{2}-2 \lambda_{345}\right) \cos 2 \beta\right), \\
M_{h S}^{\rho} & =\frac{v v_{S}}{2}\left(\lambda_{11 S}+\lambda_{22 S}+\cos 2 \beta\left(\lambda_{11 S}-\lambda_{22 S}\right)+2 \lambda_{12 S} \sin 2 \beta\right), \\
M_{H H}^{\rho} & =\sec ^{2} \beta\left(M_{22}^{2}+\frac{\lambda_{2}}{2} v^{2}+\frac{\lambda_{22 S}}{2} v_{S}^{2}\right)+\frac{\lambda_{1}-\lambda_{2}}{4} v^{2}-\frac{\cos ^{2} 2 \beta}{4}\left(\lambda_{1}+\lambda_{2}-2 \lambda_{345}\right) v^{2}, \\
M_{H S}^{\rho} & =\frac{v v_{S}}{2}\left(\sin 2 \beta\left(\lambda_{22 S}-\lambda_{11 S}\right)+2 \lambda_{12 S} \cos 2 \beta\right),
\end{aligned}
$$

\footnotetext{
${ }^{3}$ This is true for models with scalar mixing, but not for a pseudoscalar model, as the pseudoscalar gets no vev.
} 


$$
M_{S S}^{\rho}=2 \lambda_{S} v_{S}^{2}
$$

and we have defined $\lambda_{345}=\lambda_{3}+\lambda_{4}+\lambda_{5}$.

We shall now impose the alignment limit $[57,58]$ in the context of this model, as this is the experimentally favoured configuration in which one of the scalars couples like the SM Higgs boson. In a standard $2 \mathrm{HDM}$, the definition of alignment limit is that $M_{h H}^{\rho}=0$ so that both $h$ and $H$ are mass eigenstates ${ }^{4}$. In the case of the $2 \mathrm{HDM}+\mathrm{S}$, the presence of 3 mixed scalars makes the alignment limit more complicated. We shall enforce alignment by requiring that $M_{h H}^{\rho}=M_{h s}^{\rho}=0$, which implies

$$
\begin{aligned}
\lambda_{3} & =\frac{1}{2}\left(\lambda_{1}+\lambda_{2}-2 \lambda_{4}-2 \lambda_{5}+\left(\lambda_{1}-\lambda_{2}\right) \sec 2 \beta\right), \\
\lambda_{11 S} & =-\tan \beta\left(2 \lambda_{12 S}+\lambda_{22 S} \tan \beta\right),
\end{aligned}
$$

and sets the $h$ in Eq. (3.9) to be the mass eigenstate which corresponds to the SM Higgs, with $M_{h}=M_{h h}^{\rho}$. While some deviation from exact alignment would be compatible with current Higgs measurements, we adopt this approximation for simplicity. The remaining $2 \times 2$ matrix can be diagonalized to obtain mass eigenstates $\left\{S_{1}, S_{2}\right\}$, with eigenvalues and mixing angle given by

$$
\begin{aligned}
M_{S_{1,2}}^{2} & =\frac{1}{2}\left(M_{A}^{2}+\lambda_{5} v^{2}+\left(\lambda_{2} v^{2}-M_{h}^{2}\right) \tan ^{2} \beta\right)\left(1 \pm \frac{1}{\cos 2 \theta}\right)+\lambda_{S} v_{S}^{2}\left(1 \mp \frac{1}{\cos 2 \theta}\right), \\
\tan 2 \theta & =\frac{4 v \cos ^{2} \beta v_{S}\left(\tan \beta \lambda_{22 S}+\lambda_{12 S}\right)}{\cos 2 \beta\left(M_{A}^{2}+M_{h}^{2}-2 \lambda_{S} v_{S}^{2}-\lambda_{2} v^{2}+\lambda_{5} v^{2}\right)+M_{A}^{2}-M_{h}^{2}-2 \lambda_{S} v_{S}^{2}+\lambda_{2} v^{2}+\lambda_{5} v^{2}} .
\end{aligned}
$$

We can now rewrite the Lagrangian in terms of the mass eigenstates using Eqs. 3.9,3.10 and

$$
\begin{aligned}
H & =\cos \theta S_{1}-\sin \theta S_{2}, \\
S & =v_{S}+\sin \theta S_{1}+\cos \theta S_{2} .
\end{aligned}
$$

\subsection{HDM+S Lagrangian}

The $2 \mathrm{HDM}+\mathrm{S}$ scenario is described by the following Lagrangian

$$
\begin{aligned}
L & =L_{\mathrm{SM}}+L_{D M}+L_{S}+L_{A, H^{+}} \\
L_{D M} & =i \bar{\chi} \not \chi-m_{\chi} \bar{\chi} \chi-y_{\chi}\left(\sin \theta S_{1}+\cos \theta S_{2}\right) \bar{\chi} \chi \\
L_{s} & =\sum \frac{1}{2} \partial_{\mu} S_{i} \partial^{\mu} S_{i}-\frac{1}{2} M_{i}^{2} S_{i}^{2}-\sum_{f=u, d, l} \epsilon^{f} \sum_{i \in f} \frac{y_{i}}{\sqrt{2}}\left(\cos \theta S_{1}-\sin \theta S_{2}\right) \bar{f}_{i} f_{i}, \\
L_{A, H^{+}} & =\frac{1}{2} \partial_{\mu} A \partial^{\mu} A-\frac{1}{2} M_{A}^{2} A^{2}+\partial_{\mu} H^{+} \partial^{\mu} H^{-}-M_{H^{+}}^{2} H^{+} H^{-}+i \sum_{f=u, d, l} \epsilon^{f} \sum_{i \in f} \frac{y_{i}}{\sqrt{2}} A \bar{f}_{i} \gamma_{5} f_{i}, \\
& -\left(\sum_{i \in u, j \in d}\left(y_{i} V_{i j} \epsilon^{u} P_{L}+y_{j} V_{i j} \epsilon^{d} P_{R}\right) \bar{u}_{i} d_{j} H^{+}+\sum_{i \in l^{-}} y_{i} \epsilon_{i}^{L} \bar{\nu}_{i} P_{R} l_{i} H^{+}+h . c .\right)
\end{aligned}
$$

\footnotetext{
${ }^{4}$ In $2 \mathrm{HDM}$, the alignment limit can arise naturally in the presence of a softly broken CP2 symmetry [59-62] that imposes $\lambda_{1}=\lambda_{2}=\frac{1}{2} \lambda_{345}$ (or $\lambda_{1}=\lambda_{2}=\lambda_{345}$ for the normalization used in this paper). However, in the presence of the additional singlet we are not aware of the existence of any symmetry that can make this alignment to arise naturally. Nonetheless, we enforce it by requiring the non-diagonal entries of the first row/column of Eq. (3.11) to vanish.
} 
where $V=W, Z$, and we have neglected to write down the 3 and 4 point scalar interactions, and 4 point $S_{i}^{2} V^{2}$ interactions. The parameters $\epsilon^{f}$ depend upon the choices made for the Yukawa couplings to the two doublets, and will be discussed in detail in the following subsections. They are given in Table 1 for a number of common 2HDMs Yukawa structures.

For the purpose of DM phenomenology, the important terms in the Lagrangian are the coupling of the DM and SM fermions to the two mixed scalars, $S_{1,2}$. These terms have a similar form to the $\mathrm{H}+\mathrm{S}$ model of section 2, but now neither mediator is the SM Higgs, and greater coupling freedom has been obtained. The price we pay for this additional freedom is the introduction of flavour changing interactions. While $L_{s}$ and $L_{D M}$ are the relevant terms for direct detection and collider mono-jet searches, the full Lagrangain should be used if one wishes to impose complementary limits (like mono-W/Z, mono-Higgs, heavy resonances). Flavour constraints will arise primarily from $L_{A, H^{+}}$.

If there is some hierarchy between the 2 scalar masses (for example, $M_{2} \sim 5 \mathrm{TeV}, M_{1} \sim$ $500 \mathrm{GeV}$ ), interactions will be dominated by the exchange of the lighter scalar, while the heavier one can be approximately decoupled. In this limit we reproduce the structure of the singlet scalar model, from a gauge invariant framework, while retaining additional coupling freedom. In general, both scalar mediators will need to be retained.

\subsection{Yukawa structure}

The Yukawa interactions of the SM fermions with the Higgs doublets can be expressed as

$$
L_{\text {Yukawa }}=-\sum_{n=1,2}\left(Y_{n, i j}^{U} \bar{Q}_{L}^{i} u_{R}^{j} \widetilde{\Phi}_{n}+Y_{n, i j}^{D} \bar{Q}_{L}^{i} d_{R}^{j} \Phi_{n}+Y_{n, i j}^{L} \bar{L}_{L}^{i} l_{R}^{j} \Phi_{n}+\text { h.c. }\right) .
$$

As in standard 2HDMs, we shall need to choose Yukawa structures that keep potentially dangerous flavour violating processes under control. We outline the possibilities below, and explore the dark matter phenomenology of these choices in section 4 by determining direct detection constraints.

\subsubsection{Type I,II,X and $\mathrm{Y}$}

In $2 \mathrm{HDM}$ s of type I, II, X and Y, flavour violating processes are suppressed by imposing a symmetry which permits each type of fermion (up quarks, down quarks and leptons) to couple to only one of the Higgs doublets. This hypothesis is called Natural Flavour Conservation (NFC), and together with MFV guarantees that FCNCs are strongly suppressed. However, the presence of the charged scalar $H^{+}$still allows FCNCs at loop level. This places some constraints on the parameter $\tan \beta$, that is otherwise experimentally unconstrained in the Higgs-alignment limit.

In type I, all SM fermions couple to $\Phi_{2}$, while in type II the up quarks couple to $\Phi_{2}$ and the down quarks and leptons couple to $\Phi_{1}$. Type $\mathrm{X}$ and $\mathrm{Y}$ have the same quarks coupling as type I and II respectively, but the leptons couple to the opposite doublet. Therefore, at large $\tan \beta$, Type I and $\mathrm{Y}$ will be less constrained by di-lepton resonance searches than Type X and II (which, respectively, have the same quark couplings).

\subsubsection{Type III models and Minimal Flavour Violation}

In type III $2 \mathrm{HDMs}$, there is no symmetry to forbid the fermions from coupling to both doublets, and so no NFC assumption. It is well known that in type III $2 \mathrm{HDMs}$, in absence of some additional mechanism, FCNCs can arise at tree level. To avoid this we will implement Minimal Flavour Violation (MFV), adopting the most general version of MFV following [63, 64]. For the type III 


\begin{tabular}{|c|c|c|c|}
\hline Model & $\epsilon_{d}$ & $\epsilon_{u}$ & $\epsilon_{l}$ \\
\hline Type I & $\cot \beta$ & $\cot \beta$ & $\cot \beta$ \\
Type II & $-\tan \beta$ & $\cot \beta$ & $-\tan \beta$ \\
Type X & $\cot \beta$ & $\cot \beta$ & $-\tan \beta$ \\
Type Y & $-\tan \beta$ & $\cot \beta$ & $\cot \beta$ \\
Inert & 0 & 0 & 0 \\
\hline
\end{tabular}

Table 1: Values of the coefficients $\epsilon_{u, d, l}$ which correspond to models with discrete $\mathcal{Z}_{2}$ symmetries.

scenario, it makes no sense to use the basis $\Phi_{1,2}$ so we will instead work in the Higgs basis $\Phi_{h, H}$ where it will be easier to describing the couplings. Rewriting Eq. (3.28) in this basis we have

$$
L_{\text {Yukawa }}=-\sum_{n=h, H}\left(Y_{n, i j}^{U} \bar{Q}_{L}^{i} u_{R}^{j} \widetilde{\Phi}_{n}+Y_{n, i j}^{D} \bar{Q}_{L}^{i} d_{R}^{j} \Phi_{n}+Y_{n, i j}^{L} \bar{L}_{L}^{i} l_{R}^{j} \Phi_{n}+\text { h.c. }\right),
$$

where the matrices $Y_{h, i j}^{U, D, L}$ have to be the SM Yukawa matrices. Written in an arbitrary fermion basis, these are arbitrary $3 \times 3$ complex matrices that only need to reproduce the right mass eigenvalues and the CKM matrix. We can choose to work in the basis where they can be written as

$$
\begin{aligned}
Y_{h}^{U} & =V^{\dagger} D\left(y_{u}, y_{c}, y_{t}\right)=V^{\dagger} D_{U}, \\
Y_{h}^{D} & =D\left(y_{d}, y_{s}, y_{b}\right)=D_{D}, \\
Y_{h}^{L} & =D\left(y_{e}, y_{\mu}, y_{\tau}\right)=D_{L},
\end{aligned}
$$

where $D\left(x_{i}, \ldots, x_{j}\right)$ indicates a diagonal matrix where $x_{i}, \ldots, x_{j}$ are the diagonal elements. To have minimal flavour violation, we need to impose [63, 64]

$$
Y_{H}^{U}=P_{U}\left(Y_{h}^{U} Y_{h}^{U \dagger}\right) Y_{h}^{U},
$$

and similarly for $Y_{D}, Y_{L}$, where $P_{U, D, L}(x)$ are generic polynomials. Now because $\left(Y_{h}^{U} Y_{h}^{U \dagger}\right)^{n} Y_{h}^{U}=$ $V^{\dagger} D_{U}^{2 n+1}$ and $\left(Y_{h}^{D} Y_{h}^{D \dagger}\right)^{n} Y_{h}^{D}=D_{D}^{2 n+1}$, this results in the following Yukawa structure

$$
\begin{aligned}
Y_{H}^{U} & =V^{\dagger} P_{U}\left(D_{U}^{2}\right) D_{U}=V^{\dagger} D\left(\lambda_{u}, \lambda_{c}, \lambda_{t}\right), \\
Y_{H}^{D} & =P_{D}\left(D_{D}^{2}\right) D_{D}=D\left(\lambda_{d}, \lambda_{S}, \lambda_{b}\right), \\
Y_{H}^{L} & =P_{L}\left(D_{L}^{2}\right) D_{L}=D\left(\lambda_{e}, \lambda_{\mu}, \lambda_{\tau}\right) .
\end{aligned}
$$

This Yukawa structure guarantees that it is possible to simultaneously diagonalize the masses of the fermions and their Yukawa couplings to both doublets. Note that unfortunately this structure is not RGE invariant. Identifying all the allowed coupling patterns that avoid FCNCs and are stable under quantum corrections is beyond the scope of this work. Here we simply point out that there are possible coupling patterns beyond the type I and II 2HDMs. We shall illustrate two specific type III examples below, the Aligned model and a 2-generation model. A futher type III possibility, with approximate alignment, is discussed in Appendix C.

\subsubsection{Aligned 2HDM+S}

The Aligned 2HDM $[65,66]$ is a type-III 2HDM that interpolates between Type I and II. While Type I,II models impose a symmetry to force the fermions to couple to only to one of the two doublets, 


\begin{tabular}{|c|c|c|c|}
\hline Model & $\gamma_{d}$ & $\gamma_{u}$ & $\gamma_{l}$ \\
\hline Type I & $\pi / 2-\beta$ & $\pi / 2-\beta$ & $\pi / 2-\beta$ \\
Type II & $-\beta$ & $\pi / 2-\beta$ & $-\beta$ \\
Type X & $\pi / 2-\beta$ & $\pi / 2-\beta$ & $-\beta$ \\
Type Y & $-\beta$ & $\pi / 2-\beta$ & $\pi / 2-\beta$ \\
Inert & 0 & 0 & 0 \\
\hline
\end{tabular}

Table 2: Choices of angles $\gamma_{u, d, l}$ which correspond to models with discrete $\mathcal{Z}_{2}$ symmetries.

in the Aligned model we simply assume the fermions couple to only one linear combination of $\Phi_{1,2}$, but that such linear combination can be different for $u, d, l^{5}$. We can define the linear combinations that couple to up, down quarks and leptons $\Phi_{u, d, l}$ and their orthogonal ones $\Phi_{u, d, l \perp}$ to be

$$
\left(\begin{array}{c}
\Phi_{i} \\
\Phi_{i \perp}
\end{array}\right)=\left(\begin{array}{cc}
\cos \gamma_{i} & \sin \gamma_{i} \\
-\sin \gamma_{i} & \cos \gamma_{i}
\end{array}\right)\left(\begin{array}{c}
\Phi_{h} \\
\Phi_{H}
\end{array}\right),
$$

where $i=u, d, l$ and $0 \leq \gamma_{u, d, l}<\pi$ are arbitrary angles ${ }^{6}$. By requiring the fermion interactions with the Higgs are the same as in the SM, we identify the coupling to the 2nd doublet

$$
\begin{aligned}
& Y_{h}^{U} \equiv Y_{\mathrm{SM}}^{U}, \\
& Y_{H}^{U}=\tan \gamma_{u} Y_{\mathrm{SM}}^{U},
\end{aligned}
$$

and similarly for $d, l$. With this identification, the fermion couplings to the new doublet differ from the SM Yukawa couplings by an overall scaling factor, $\tan \gamma_{i}$, where $\gamma_{i}$ can take different values for $u, d, l$. This arrangement clearly satisfies the simultaneously diagonalizable requirement of type III models that respect MFV. One can observe that in principle it is possible for the $b$-quark to couple more strongly to the $S_{1,2}$ mediators than the $t$-quark (this is true also for Type II with large $\tan \beta$ ) which suggests the possibility of different experimental signatures. In fact, $2 \mathrm{HDM}$ of type I, II, X and Y can be recovered with particular choices for the angles $\gamma_{i}$, as listed in Table 2.

\subsubsection{Coupling to only the first 2 generations (2gen-2HDM+S)}

Until now, all our models have had couplings to quarks that are proportional to the SM Yukawa couplings. As a consequence the $t$ and $b$ quark couplings will be dominant, as all other couplings will be suppressed by a factor of at least $\frac{m_{s}}{m_{b}}$. Thus all the relevant collider phenomenology will be dominated by initial states with gluons and maybe $b$ quarks, and only final states with $g, b$ jets or top quarks. On the other hand, most flavour constraints that enforce this Yukawa structure are coming from the couplings to the third generation of quarks. Thus it might be interesting to understand what happens to flavour constraints when one turns off the couplings to the third family of quarks. Logically, in such case the phenomenology will be completely different, as $b, t$ will not be present in the initial and final states, and also $g g$ initial states with top loop diagrams will no longer contribute.

\footnotetext{
${ }^{5}$ Note that this hypothesis is not stable under quantum corrections, so the model will not be NFC compliant, while still MFV. Loop corrections have been studied in several works [67-69] and found not to be tightly constraining.

${ }^{6}$ References (e.g. [66, 70, 71]) define the Aligned model in terms of the parameters $\zeta_{u, d, l}$, that can be complex in general. Imposing CP conservation forces them to be real and in such a case they are related to the $\gamma_{i}$ parameters above by $\zeta_{i}=\tan \left(\gamma_{i}\right)$
} 
Ref. [69] analyses in detail the possible Yukawa structures that can lead to stable RGE solutions. While they focus on solutions that satisfy the Yukawa alignment condition $Y_{H}^{i} \propto Y_{h}^{i}, i=u, d$, the solution 5 that they find in the appendix is a viable solution also for $Y_{i}^{u} \propto Y_{i}^{d}, i=H, h$. Therefore, a possible Yukawa structure for the additional doublet that may avoid flavour constraints is ${ }^{7}$

$$
\begin{aligned}
Y_{H}^{U} & =A V^{\dagger} P_{12}, \\
Y_{H}^{D} & =B P_{12},
\end{aligned}
$$

where $A, B$ are real numbers and

$$
P_{12}=\left(\begin{array}{lll}
1 & 0 & 0 \\
0 & 1 & 0 \\
0 & 0 & 0
\end{array}\right), \quad P_{3}=\left(\begin{array}{lll}
0 & 0 & 0 \\
0 & 0 & 0 \\
0 & 0 & 1
\end{array}\right) .
$$

The new doublet therefore couples to the first 2 generations of quarks only, with equal strength. We neglect the leptons for convenience, as they can always be taken to be flavour diagonal (or zero) such that no FCNCs arise in the lepton sector. This structure has been chosen because it is orthogonal to the one of the SM Yukawa matrices, that, in the same basis, are

$$
\begin{aligned}
& Y_{h}^{U}=y_{t} V^{\dagger} P_{3}+O\left(\frac{m_{c}}{m_{t}}\right), \\
& Y_{h}^{D}=y_{b} P_{3}+O\left(\frac{m_{s}}{m_{t}}\right) .
\end{aligned}
$$

This is a convenient form ${ }^{8}$ that strongly suppresses FCNCs, as they must be proportional either to one of the small parameters $y_{u}, y_{d}, y_{s}, y_{c}$ or to at least one of the small CKM matrix elements, $V_{13}, V_{23}, V_{31}, V_{32}$. We can check that this Yukawa structure doesn't induce large FCNC, provided $A \lesssim 0.1$ and $B \lesssim 0.01$, by solving the RGE using the formalism of [76, 77]. This is done in Appendix B. Note the this structure can therefore be used to obtain couplings for $c, s, d, u$ that, respectively, are up to 13,18,400, 7500 times larger than in the SM. Given these enhanced couplings, we therefore expect these models will be subject to much tighter direct detection constraints, unless some relative cancellation or interference occurs.

\section{Direct Detection}

Direct Detection (DD) is an efficient way of probing these models. Interactions mediated by the exchange of scalar particles generate Spin-Independent operators (SI) that can currently probe cross sections of the order of $10^{-45} \mathrm{~cm}^{2}$ for DM particles in the range $10 \mathrm{GeV}<m_{\chi}<1 \mathrm{TeV}$. In particular, DD will typically be more efficient that collider searches in the high-mass range, $m_{\chi} \gtrsim 100 \mathrm{GeV}$, where collider production cross sections are significantly suppressed. However, DD will usually leave open a small window at low $\left(m_{\chi} \lesssim 10 \mathrm{GeV}\right)$ DM masses. In this window, collider physics usually provides stronger constraints.

The gauge invariant class of models considered in this paper introduce another parameter region where direct detection searches are blind: When the masses of the scalar mediators are

\footnotetext{
${ }^{7}$ Yukawa patterns that can yield similar enhancements for the signal of the first 2 generations can also be found in $[72-75]$.

${ }^{8}$ Note that this structure formally respects MFV, even though it requires a fine tuning of $\mathcal{O}\left(\frac{y_{u}}{y_{c}}, y_{c}^{2}\right)$.
} 
similar, interference effects will be very important[78-80]. This feature will be present in all the models we considered (in both the $\mathrm{H}+\mathrm{S}$ and $2 \mathrm{HMD}+\mathrm{S}$ scenarios). In fact, for degenerate mass mediators, the DD nucleon operators cancel exactly. Since this cancellation is not accidental, but rather arises from a symmetry, it should be expected to hold at loop level. 1-loop corrections were calculated for this model and were indeed found to have negligible contributions to the tree level results presented in this paper. In the case of $2 \mathrm{HDM}+\mathrm{S}$, there will be an additional source of interference: the interference between different quarks present in nucleons. For example, in Type II 2HDMs with Higgs alignment, the up and down quarks have Yukawa couplings of opposite sign, which will lead to opposite-sign contributions to the coefficient of the effective nucleon operator.

\subsection{Direct detection constraints for the $\mathrm{H}+\mathrm{S}$ model}

This model will be strongly constrained by DD experiments, as the $125 \mathrm{GeV}$ Higgs is one of the mediators. Unless the SM Higgs and second mediator masses are approximately degenerate, interference will play no role and the DD cross section will be dominated by the exchange of the SM Higgs, placing a very strong bound on the value of the mixing angle $\epsilon$. The only relevant nucleon operator will be

$$
O_{1}^{N}=\bar{\chi} \chi \bar{N} N
$$

with a coefficient related to those for the quark and gluon operators

$$
\begin{aligned}
& O_{1}^{q}=\bar{\chi} \chi \bar{q} q, \\
& O_{1}^{g}=\frac{\alpha_{s}}{12 \pi} G_{a}^{\mu \nu} G_{\mu \nu}^{a} \bar{\chi} \chi,
\end{aligned}
$$

according to

$$
c_{N}=\sum_{q=u, d, s} \frac{m_{N}}{m_{q}} f_{T_{q}}^{N} c_{q}+\frac{2}{27} f_{T_{g}} \sum_{q=c, b, t} \frac{m_{N}}{m_{q}} c_{q} .
$$

Starting from the Lagrangian at a high energy, we can evolve down to low energies, integrating out first $s$ and then $h$, to generate the following EFT operator for light quarks:

$$
O_{q}=c_{q} O_{1}^{q}=\frac{y_{q} y_{\chi} \cos \epsilon \sin \epsilon}{\sqrt{2}}\left(\frac{1}{M_{h}^{2}}-\frac{1}{M_{S}^{2}}\right) \bar{\chi} \chi \bar{q} q .
$$

Heavy quarks instead contribute through the gluon operator:

$$
O_{g}=-\frac{c_{q}}{m_{q}} O_{1}^{g}=-\frac{c_{q}}{m_{q}} \frac{\alpha_{s}}{12 \pi} G_{a}^{\mu \nu} G_{\mu \nu}^{a} \bar{\chi} \chi
$$

The coefficient of the nucleon operator 4.1 is thus

$$
c_{N}=m_{N} \frac{y_{\chi} \cos \epsilon \sin \epsilon}{v}\left(\frac{1}{M_{h}^{2}}-\frac{1}{M_{S}^{2}}\right)\left(\sum_{q=u, d, s} f_{T_{q}}^{N}+\frac{2}{9} f_{T_{g}}\right) .
$$

Note that in this scenario, interference effects between different quarks are not possible as all fermion couplings to the new scalar are proportional to the Yukawa couplings to the SM Higgs (with the same proportionality coefficient) $)^{9}$.

\footnotetext{
${ }^{9}$ This will be true also for Type I $2 \mathrm{HDM}+\mathrm{S}$, where the same situation applies.
} 

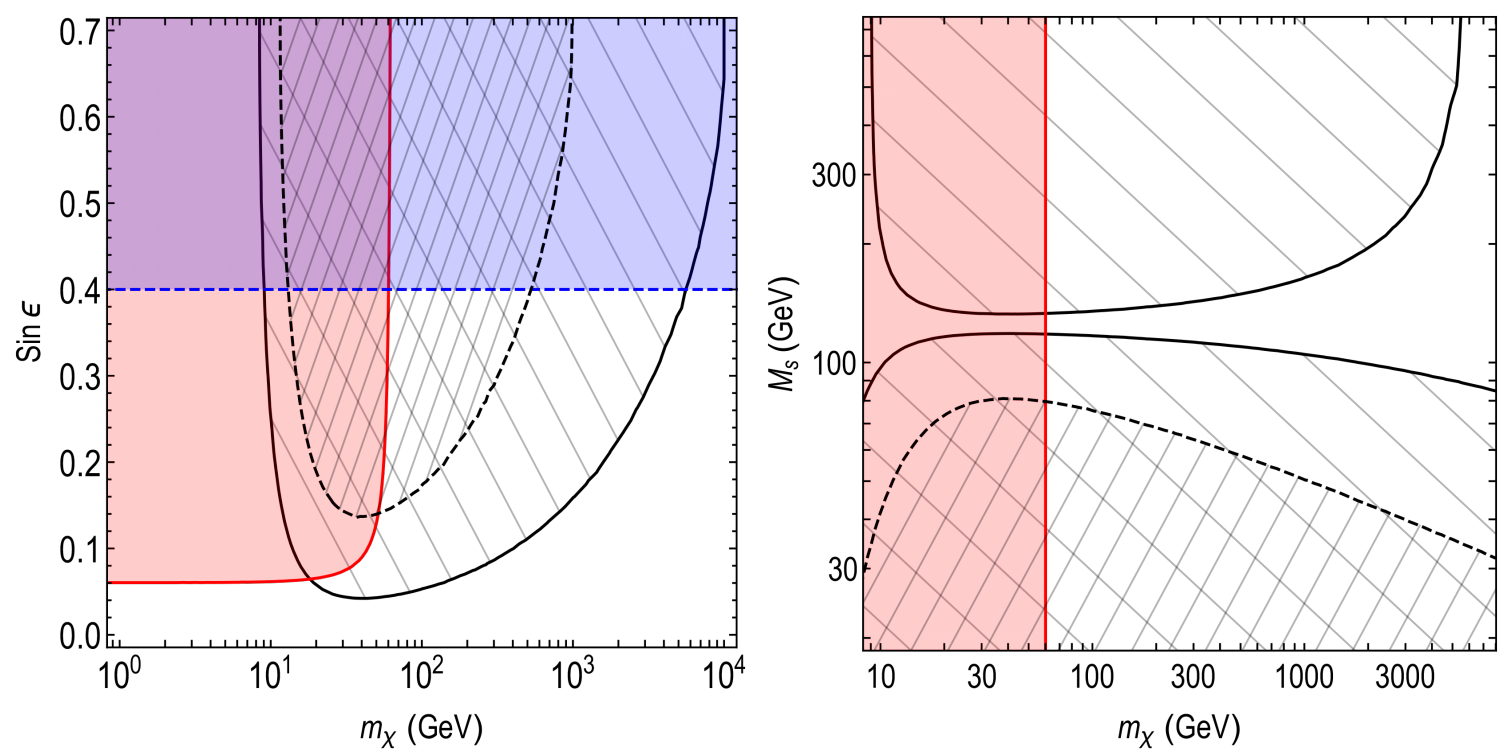

Figure 3: Direct detection limits on the $\mathrm{H}+\mathrm{S}$ model, using LUX data. Left panel: limits on the mixing angle $\epsilon$ as a function of DM mass for fixed values of the mass of the second mediator, for $y_{\chi}=1$. The solid black line refers to a heavy mediator $\left(M_{S}=1 \mathrm{TeV}\right)$, while the dashed line refers to a mediator nearly degenerate with the SM Higgs $\left(M_{S}=150 \mathrm{GeV}\right)$. The red region is excluded by Higgs invisible width constraints, while the blue region is excluded by precision electroweak constraints on $\sin \epsilon$ from [27]. Right panel: limits on the mass of the second mediator $M_{S}$ as a function of the dark matter mass for fixed values of the mixing angle. The solid black line refers to $\sin \epsilon=0.4$, while dashed black line refers to $\sin \epsilon=0.03$. The red region is excluded by the Higgs invisible width for $\sin \epsilon=0.4$; there is no corresponding bound on $m_{\chi}$ for $\sin \epsilon=0.03$.

DD constraints for these models are generated using tools from [81]. The left panel of Fig. 3 shows limits from LUX [82] on the mixing parameter $\epsilon$ as a function of the DM mass for $y_{\chi}=1$. The solid lines assume a heavy scalar mediator $\left(M_{S}=1 \mathrm{TeV}\right)$, while the dashed lines refer to a nearly degenerate scalar of $M_{S}=150 \mathrm{GeV}$. The region excluded by invisible Higgs decays is also shown (red shaded region), which is complementary to DD, covering the low DM mass region. Finally, the blue shaded region refers to $\sin \epsilon>0.4$, which is excluded by EW precision data [27]. In the right panel we instead fix the mixing angle to be $\sin \epsilon=0.4$ for the solid lines, and $\sin \epsilon=0.03$ for the dashed lines, and show limits on $M_{S}$ as a function of the DM mass. For $\sin \epsilon=0.4$, one can see that there is a very large range of DM masses for which only the nearly-degenerate scenario is allowed. This permits the second mediator mass to lie close to that of the $125 \mathrm{GeV}$ Higgs, with larger and smaller values both excluded. This is because the nucleon operator coefficient in eq. (4.7) is very large when mediated by the Higgs boson - hence to avoid DD constraints, we require the second scalar to have a similar mass in order to have strong cancellation between the two diagrams. For such a mixing angle, the low DM mass mass region, $m_{\chi} \lesssim M_{h} / 2$, is excluded by Higgs invisible decays (red shaded). For a much smaller scalar mixing angle, $\sin \epsilon=0.03$, the upper bound on the mass of the second mediator disappears, opening up the heavier-mediator region. Note that, as in this plot we are fixing the mixing angle to some fixed value, the perturbativity of the coupling $\lambda_{H S}$ together with eq. (2.6) will necessarily give an upper bound on the mass of the new scalar. Because of this, it's never possible to completely decouple the second scalar by taking the limit $M_{S} \rightarrow \infty$ while keeping the mixing angle value fixed. 


\subsection{Direct detection constraints for $2 \mathrm{HDM}+\mathrm{S}$ scenarios}

The direct detection formalism for this case is similar to the preceding one, however the phenomenology is different because the mass of the first scalar is not fixed to be the SM Higgs mass and the mixing angle is unconstrained from SM physics. Moreover, the Yukawa couplings of the second doublet are no longer forced to all be rescaled by the same factor, and we will check the effect of the non-standard Yukawa patterns, as described in the previous sections, including destructive interference between different quarks types.

The quark and gluon operators are now

$$
\begin{aligned}
& O_{q}=c_{q} O_{1}^{q}=\frac{\lambda_{q} y_{\chi} \cos \theta \sin \theta}{\sqrt{2}}\left(\frac{1}{M_{S_{1}}^{2}}-\frac{1}{M_{S_{2}}^{2}}\right) \bar{\chi} \chi \bar{q} q, \\
& O_{g}=-\frac{c_{q}}{m_{q}} O_{1}^{g},
\end{aligned}
$$

where the value of $\lambda_{q}$ depends on the specific model. For the specific Yukawa structures discussed in section 3.3, we obtain the following coefficients for the nucleon operator:

$$
\begin{aligned}
c_{N}^{\text {type I }} & =m_{N} \frac{y_{\chi} \cos \theta \sin \theta}{v \tan \beta}\left(\frac{1}{M_{S_{1}}^{2}}-\frac{1}{M_{S_{2}}^{2}}\right)\left(\sum_{q=u, d, s} f_{T_{q}}^{N}+\frac{2}{9} f_{T_{g}}\right) \\
c_{N}^{\text {type II }} & =m_{N} \frac{y_{\chi} \cos \theta \sin \theta}{v}\left(\frac{1}{M_{S_{1}}^{2}}-\frac{1}{M_{S_{2}}^{2}}\right)\left(f_{T_{u}}^{N} \cot \beta-\tan \beta \sum_{q=d, s} f_{T_{q}}^{N}+\frac{2}{9} f_{T_{g}} \frac{2 \cot \beta-\tan \beta}{3}\right) \\
c_{N}^{\text {aligned }} & =m_{N} \frac{y_{\chi} \cos \theta \sin \theta}{v}\left(\frac{1}{M_{S_{1}}^{2}}-\frac{1}{M_{S_{2}}^{2}}\right)\left(f_{T_{u}}^{N} \tan \gamma_{u}+\tan \gamma_{d} \sum_{q=d, s} f_{T_{q}}^{N}+\frac{2}{9} f_{T_{g}} \frac{\tan \gamma_{u}+2 \tan \gamma_{d}}{3}\right) \\
c_{N}^{\text {2gen }} & =m_{N} \frac{y_{\chi} \cos \theta \sin \theta}{v}\left(\frac{1}{M_{S_{1}}^{2}}-\frac{1}{M_{S_{2}}^{2}}\right)\left(A\left(\frac{f_{T_{u}}^{N}}{y_{u}}+\frac{2 f_{T_{g}}}{27 y_{c}}\right)+B \sum_{q=d, s} \frac{f_{T_{q}}^{N}}{y_{q}}\right)
\end{aligned}
$$

One can indeed note the presence of negative interference in the Type II scenario, and the possibility to also achieve it in the Aligned and 2-generation models, for appropriate values of $\gamma_{u, d}$ and $\{A, B\}$, respectively.

Fig. 4 shows the DD limits for $\theta=\pi / 4$ for Type I (upper left panel), Type II (upper right panel), aligned (lower left panel) and 2-gen (lower right panel) 2HMD+S scenarios. For Type I and II we choose $\tan \beta=1$ or 1,10 respectively, while for the aligned and 2-gen cases we choose different values of $\gamma_{u, d}$ and $A, B$ respectively (including opposite signs). Different value of $M_{S_{2}}$ were chosen in each case, as labelled on the figure caption. A general feature of these results is the presence of not only a lower bound, but also of an upper bound on $M_{S_{1}}$, for fixed values of $M_{S_{2}}$ below a certain threshold value.

This feature was present also in the $\mathrm{H}+\mathrm{S}$ model, and its origin is the same; when one of the two mediators has a mass that is too low and would be excluded by DD constraints - if taken alone - one can always evade DD constraints by taking the other mediator to be nearly degenerate, thus allowing a cancellation in the nucleon operator coefficients in eq. (4.10, 4.11, 4.12, 4.13). As before, in these plots we are fixing the mixing angle and the mass of one of the scalars, thus setting an upper bound on the mass of the second scalar due to perturbativity. In this case, however, the upper bound can be increased by having a sufficiently large enough singlet vev $v_{s}$. 

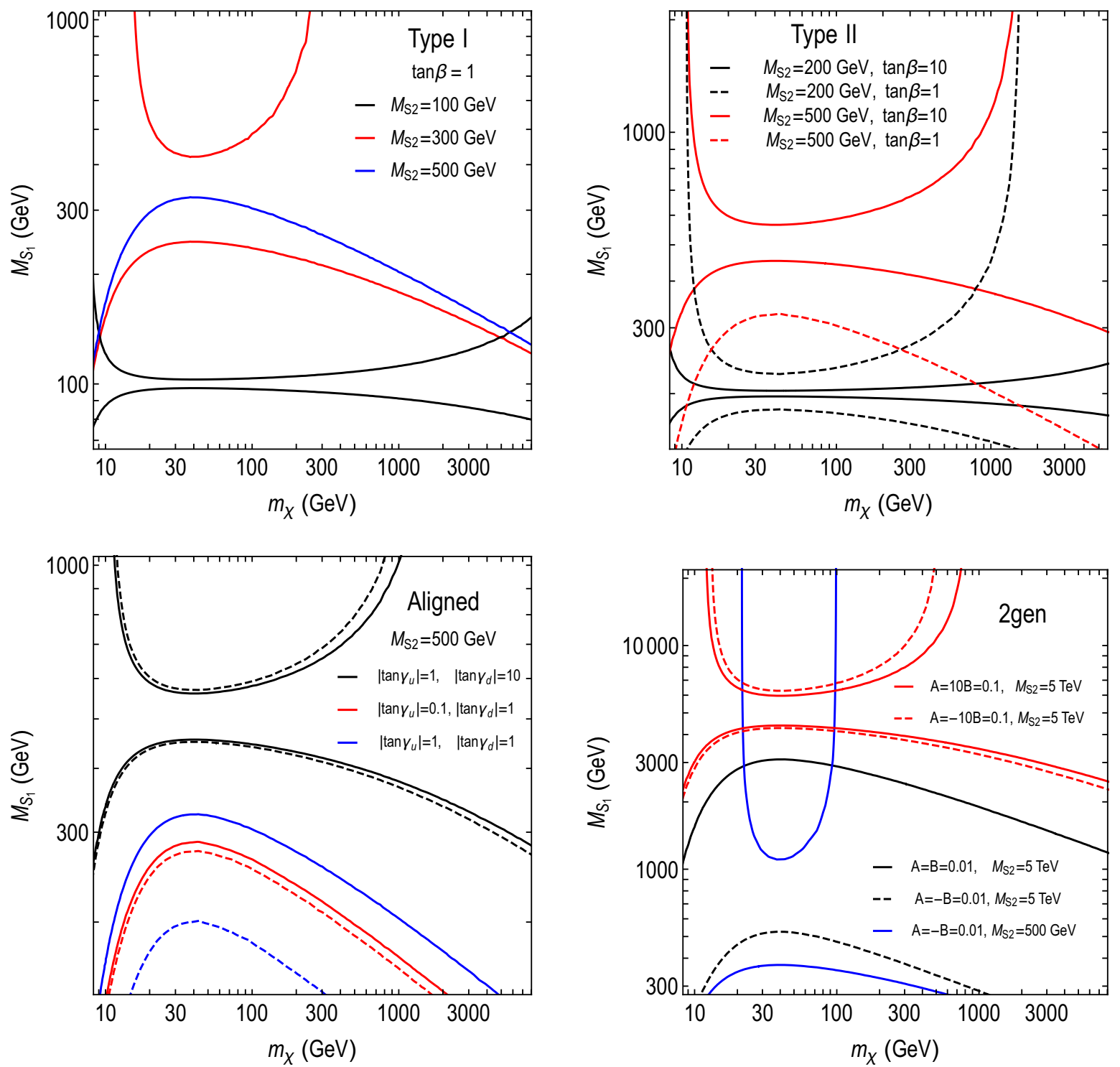

Figure 4: Direct detection limits for Type I, Type II, Aligned and 2gen 2HDMs, using LUX data. All panels assume maximal mixing between the 2 additional scalars $(\theta=\pi / 4)$. For the Aligned model, the solid and dashed curves denote refer to $\tan \gamma_{u}$ and $\tan \gamma_{d}$ of the same or opposite sign, respectively. For the 2-gen model with $A=B=0.01$, $M_{S 2}=500 \mathrm{GeV}$ or $A= \pm 10 B=0.1, M_{S 2}=500 \mathrm{GeV}$, all the parameter space is excluded except for the very degenerate region $M_{S 1} \approx M_{S 2}$, and so was not plotted.

In Type I, if one of the two mediators is light, $M_{S_{2}} \lesssim 100 \mathrm{GeV}$, only the nearly-degenerate scenario is allowed, while progressively increasing the mass of one of the scalar opens up more parameter space in the plane $M_{S_{1}}-m_{\chi}$. For $M_{S_{2}}=300 \mathrm{GeV}$, one still has an upper bound on $M_{S_{1}}$ in a certain range of DM masses. Only when $M_{S_{2}} \gtrsim 400 \mathrm{GeV}$ is the other mediator allowed to be arbitrarily heavy for any DM mass. In Type II we note the effect of interference for $\tan \beta=1$ and a fixed value of $M_{S_{2}}$. While in Type I increasing the value of $\tan \beta$ leads to weaker limits, for Type II we have the opposite situation, and this is not only because the coupling to down quarks increases, but also because we have an important interference effect for $\tan \beta \lesssim 1$. For both the mediator 
masses shown, $M_{S_{2}}=200 \mathrm{GeV}, 500 \mathrm{GeV}$ (black, red), the choice $\tan \beta=1$ (dashed) always results in weaker limits than $\tan \beta=10$ (solid).

An interference effect also arises in the aligned model, where we have more freedom with the couplings. In this case interference arises when $\tan \gamma_{d} \cot \gamma_{u}<0$. The coefficient in Eq. (4.12) cancels when $\tan \gamma_{d}$ and $\tan \gamma_{u}$ are of similar magnitude but opposite sign. Of the cases displayed, we can indeed notice that interference plays an important role only for $\tan \gamma_{u}=-\tan \gamma_{d}= \pm 1$ (blue curves). Finally, in the model were we couple only the first two generations with equal couplings, we no longer have contributions from $t, b$ quarks, but light quarks contributions can be significantly enhanced. Choosing couplings $A=0.1, B=0.01$ results in more than an order-of-magnitude increase for the limits on the scalar mediator mass, compared with the preceding cases. In this model, we can see that interference plays an important role only if $A \sim-B$.

\section{Conclusions}

We have considered the class of models in which a fermion DM candidate, uncharged under the SM gauge group, interacts with SM fermions via the exchange of an $s$-channel scalar mediator. The single-mediator Simplified Model version of this scenario is not gauge invariant. Instead, this scenario can be realised via the mixing of a singlet scalar which couples to DM, with an $S U(2)_{L}$ doublet scalar which couples to SM fermions. If this $S U(2)_{L}$ doublet scalar is none other than the SM Higgs, this leads to a situation where all couplings of quarks and leptons to the two mixed scalars are proportional to the SM Yukawa couplings, with a universal proportionality constant set by the scalar mixing angle.

Alternatively, the singlet scalar may mix with an additional SU(2) doublet in a two Higgs doublet model. This permits much greater freedom for the masses and mixing of the scalar mediators, and their Yukawa couplings to SM fermions. In this case, the proportionality constant can be made sector dependent, taking different values for the $u$-quark, $d$-quark and lepton sectors. For example, it is possible to enhance the coupling to $d$-type quarks, suppress the coupling to top-quarks, or obtain a leptophilic or leptophobic model. Moreover, it is possible to obtain flavour dependent couplings without inducing large FCNCs. Such Yukawa structures would lead to very different collider phenomenology.

We calculated direct detection constraints on the $\mathrm{H}+\mathrm{S}$ and $2 \mathrm{HDM}+\mathrm{S}$ models, which both feature spin-independent DM-nucleon scattering. Much of the parameter space of the $\mathrm{H}+\mathrm{S}$ model is excluded by direct detection data, unless either the scalar mixing angle is very small, or the mass of the new scalar is approximately degenerate with the SM Higgs, suppressing the scattering cross section via destructive interference. For the $2 \mathrm{HDM}+\mathrm{S}$ model, interference of the two scalar mediators is again very important. Moreover, an additional source of interference is now provided by relative cancellations between different quarks in the nucleus. This occurs when up and down type quarks have Yukawa couplings of opposite sign, as can occur in the Type II, Aligned and 2-generation Yukawa structures we examined. As a result, unlike the $\mathrm{H}+\mathrm{S}$ model, the $2 \mathrm{HDM}+\mathrm{S}$ scenario has substantial parameter space that is not eliminated by direct detection.

It is clear that the phenomenology of these minimal self consistent scenarios (both $\mathrm{H}+\mathrm{S}$ and $2 \mathrm{HDM}+\mathrm{S}$ ) is much richer than that of the single-mediator (non gauge invariant) Simplified Model. If there is a hierarchy of scalar masses, such that the heaviest decouples and interactions are dominated by the exchange of the lightest, we recover the structure of the single-mediator model from a gauge 
invariant framework. In general, all three scalars of the $2 \mathrm{HDM}+\mathrm{S}$ scenario would mix. However, we took a generalised Higgs alignment limit, where the SM Higgs decouples and the singlet mixes only with the additional doublet. Experimental constraints on Higgs properties require that this limit is at least approximately realised, though there would be scope to relax this assumption while still abiding by current Higgs constraints. Regardless, it is evident that the $s$-channel scalar mediator scenario should in general be analysed in a multi-mediator context, with a minimum of two scalars.

\section{Acknowledgements}

This work was supported in part by the Australian Research Council. We thank Yi Cai, Matthew Dolan, Francesco D'Eramo and Gino Isidori for helpful discussions, and Ulrich Haisch for detailed comments on an earlier draft of the manuscript. Feynman diagrams were drawn using TikZFEYNMAN [83].

\section{A Unitarity, Stability and perturbativity of $2 \mathrm{HDM}+\mathrm{S}$}

\section{A.1 Unitarity and perturbativity}

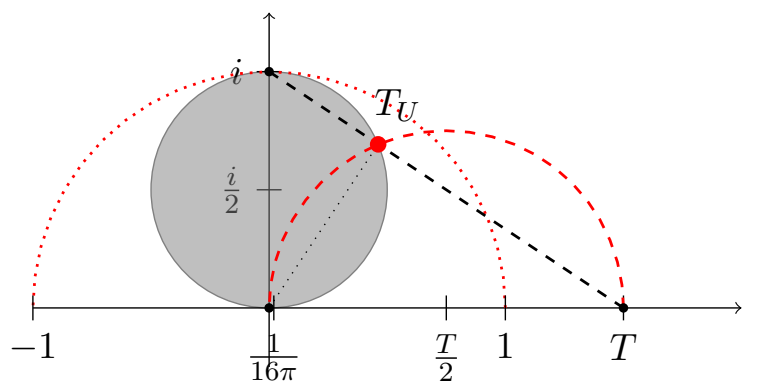

Figure 5: Argand circle and Thales projection $T_{U}$.

We start with the scattering amplitude $M$ for the scalar particles,

$$
M_{\Phi_{i} \Phi_{j} \rightarrow \Phi_{k} \Phi_{l}}=\frac{\partial^{4} V}{\partial \Phi_{i} \partial \Phi_{j} \partial \Phi_{k}^{\dagger} \partial \Phi_{l}^{\dagger}} .
$$

This matrix is hermitian, so its eigenvalues are real. The $T$-matrix is related to it by

$$
T=\frac{1}{16 \pi} M
$$

The unitarity of the model is satisfied as long as the eigenvalues of $T$ satisfy $^{10}$

$$
\mid \text { Eigenvalues }[T] \mid \leq 1
$$

This condition means that the scattering amplitude at tree level does not exceed the unitarity limit for the cross section. Looking at the Argand circumference in Fig. 5, this means that the amplitude

\footnotetext{
${ }^{10}$ See [84] for more details.
} 
$T$ lies inside the red dotted circumference of radius 1 . If, however, we also want perturbativity, then we need to impose a more stringent condition [85]

$$
\mid \text { Eigenvalues }[M]|\leq \xi \rightarrow| \text { Eigenvalues }[T] \mid \leq \frac{\xi}{16 \pi},
$$

where the choice of a maximum value of $\xi=1,2$ is somehow arbitrary, and this condition means that our amplitude, that is real and so is lying on the real axes, is not far away from the unitarity circle, and then the loop corrections necessary to move it inside it are small compared to the tree level amplitude ${ }^{11}$.

The scattering matrix $M$ is block diagonal, the blocks being related to initial and final states of same electric charge. The charge 1 block can be put in block diagonal form as

$$
\begin{aligned}
A_{0+\rightarrow 0+} & =\operatorname{Block}\left[B_{1}, B_{2}, B_{3}, D\left(\lambda_{3}+\lambda_{5}, \lambda_{3}-\lambda_{5}, \lambda_{3}+\lambda_{4}, \lambda_{3}-\lambda_{4}\right)\right], \\
B_{1} & =\left(\begin{array}{cc}
\lambda_{11 S} & \lambda_{12 S} \\
\lambda_{12 S} & \lambda_{22 S}
\end{array}\right), \\
B_{2} & =\left(\begin{array}{ll}
\lambda_{1} & \lambda_{4} \\
\lambda_{4} & \lambda_{2}
\end{array}\right) \\
B_{3} & =\left(\begin{array}{ll}
\lambda_{1} & \lambda_{5} \\
\lambda_{5} & \lambda_{2}
\end{array}\right) .
\end{aligned}
$$

The charge 2 scattering matrix is instead

$$
A_{++\rightarrow++}=\operatorname{Block}\left[B_{2}, D\left(\lambda_{3}+\lambda_{4}\right)\right]
$$

From $A_{0+\rightarrow 0+}$ and $A_{++\rightarrow++}$ we get the following constraints:

$$
\begin{aligned}
\left|\lambda_{3}\right|+\left|\lambda_{4}\right| & <1, \\
\left|\lambda_{3}\right|+\left|\lambda_{5}\right| & <1, \\
\lambda_{1}+\lambda_{2}+\sqrt{\lambda_{1}^{2}-2 \lambda_{2} \lambda_{1}+\lambda_{2}^{2}+4 \lambda_{5}^{2}} & <2, \\
\lambda_{1}+\lambda_{2}+\sqrt{\lambda_{1}^{2}-2 \lambda_{2} \lambda_{1}+\lambda_{2}^{2}+4 \lambda_{4}^{2}} & <2, \\
\lambda_{11 S}+\lambda_{22 S}+\sqrt{\lambda_{11 S}^{2}+4 \lambda_{12 S}^{2}-2 \lambda_{11 S} \lambda_{22 S}+\lambda_{22 S}^{2}} & <2,
\end{aligned}
$$

where (A.12) and (A.13) can be rewritten as

$$
\lambda_{4,5}^{2}<\left(1-\lambda_{1}\right)\left(1-\lambda_{2}\right), \quad \lambda_{1,2}<1 .
$$

Regions of the parameter space where eigenvalues of $B_{2}, B_{3}$ are perturbative are shown in the left panel of Fig. 6 for $\xi=1,2$, assuming the presence of the CP2 symmetry $^{12}\left(\lambda_{1}=\lambda_{2}=\lambda_{3}+\lambda_{4}+\lambda_{5}=\right.$ $\left.\frac{M_{h}^{2}}{v^{2}}\right)$, so that no condition depends on $\beta$. The right panel instead show the regions of parameters space where eigenvalues of $B_{1}$ are perturbative, for several values of $\tan \beta$ and $\xi=1$ (note that the regions in this plot scale linearly with $\xi$ ). At large $\tan \beta$ the parameter space shrinks due to the alignment condition, and fine-tuning is necessary to guarantee the unitarity and perturbativity of all couplings. 

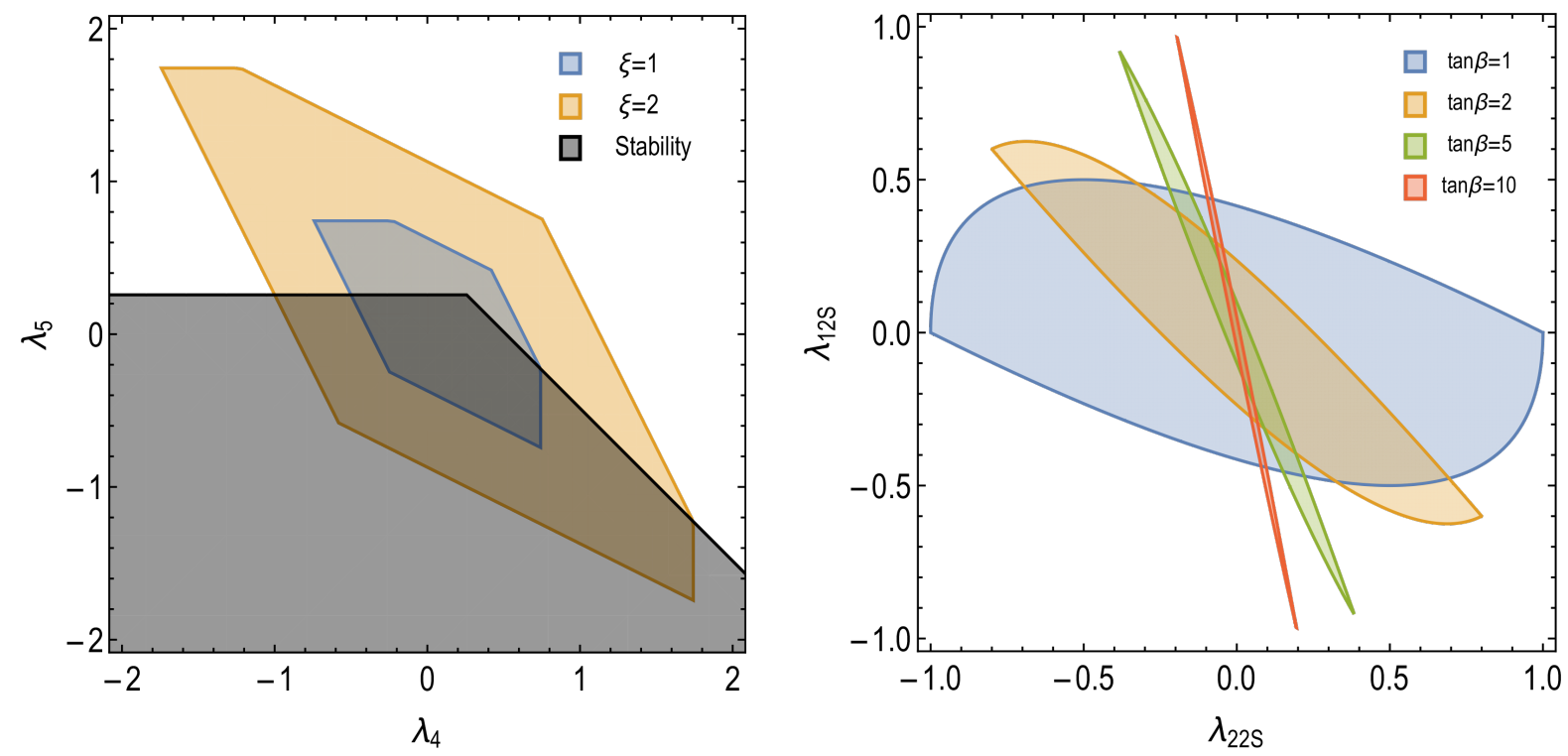

Figure 6: Regions of the parameters space satisfying perturbativity constrains from scattering matrix $A_{0+\rightarrow 0+}$. Left panel: the coloured regions are where the model is perturbative, while the black shaded region is the one excluded by global stability of the potential (CP2 symmetry assumed in this plot). Right panel: Allowed regions for $\xi=1$ for varying $\tan \beta$. Note that the dimensions of all regions in this plot scale linearly with $\xi$.
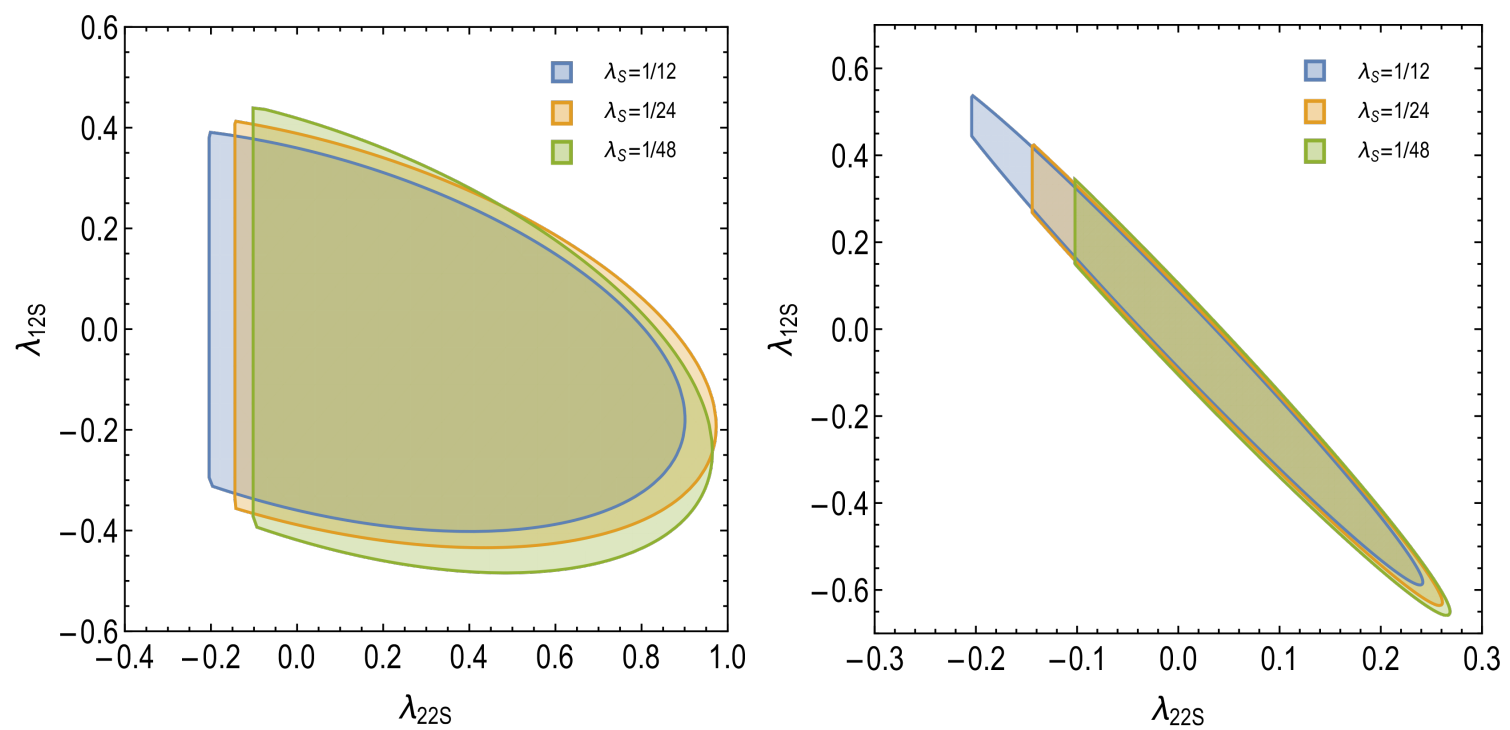

Figure 7: Regions of the parameters space satisfying perturbativity constrains from scattering matrix $A_{00 \rightarrow 00}$ for $\xi=2$. Left panel: $\tan \beta=1$, Right panel: $\tan \beta=5$. Lower limits on $\lambda_{22 S}$ come from global stability conditions.

The zero-charge scattering matrix is

$$
A_{00 \rightarrow 00}=\operatorname{Block}\left[B_{1}, B_{1}, B_{2}, B_{3}, B_{3}, B_{4}, D\left(\lambda_{3}+\lambda_{5}, \lambda_{3}-\lambda_{5}, \lambda_{3}+\lambda_{4}, \lambda_{3}-\lambda_{4}\right)\right]
$$

\footnotetext{
${ }^{11}$ Or that, in other words, the Thales projection $T_{U}$ is very close to the tree level amplitude $T$, namely $\left|T_{U}-T\right|^{2} \ll|T|^{2}$

${ }^{12}$ Note that this condition becomes $\lambda_{1}=\lambda_{2}=\frac{1}{2}\left(\lambda_{3}+\lambda_{4}+\lambda_{5}\right)$ when the normalization of the Lagrangian is taken to be the alternative of what we have chosen, as is done in [60].
} 


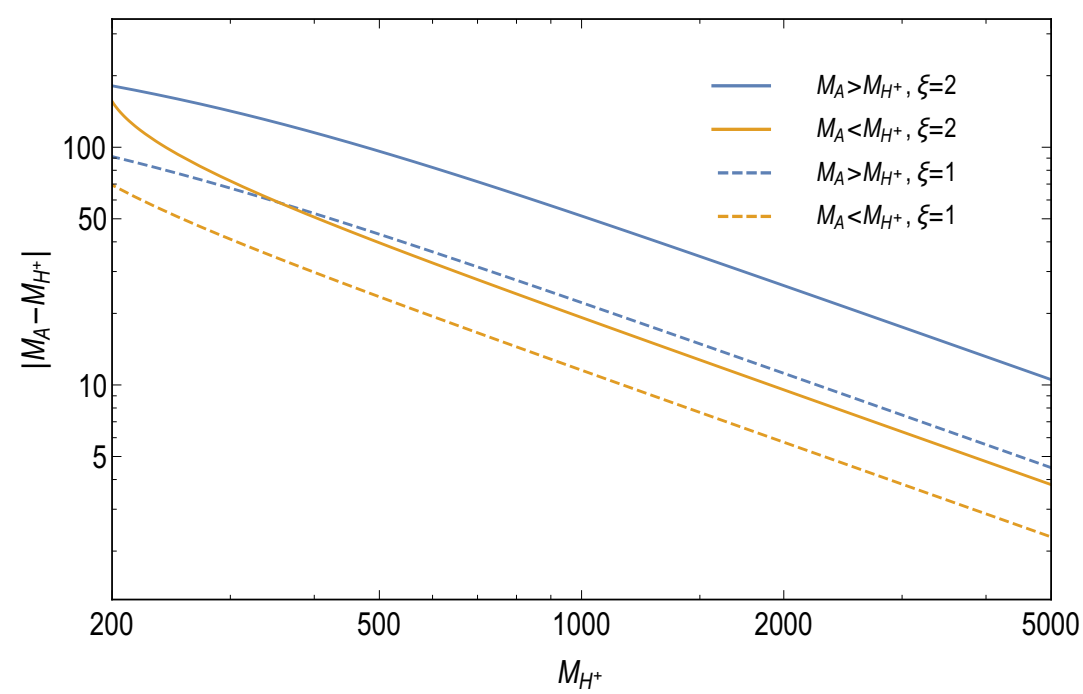

Figure 8: Maximum mass splitting between the charged scalar and the pseudoscalar for different values of $\xi$.

$$
B_{4}=\left(\begin{array}{cccc}
\lambda_{3}+2 \lambda_{4}+3 \lambda_{5} & 0 & 0 & 2 \lambda_{12 S} \\
0 & 3 \lambda_{1} & 2 \lambda_{3}+\lambda_{4} & \sqrt{2} \lambda_{11 S} \\
0 & 2 \lambda_{3}+\lambda_{4} & 3 \lambda_{2} & \sqrt{2} \lambda_{22 S} \\
2 \lambda_{12 S} & \sqrt{2} \lambda_{11 S} & \sqrt{2} \lambda_{22 S} & 6 \lambda_{S}
\end{array}\right)
$$

All eigenvalues of $A_{00 \rightarrow 00}$ except the ones of $B_{4}$ already satisfy perturbativity constraints in the regions where the matrices $A_{++\rightarrow++}, A_{0+\rightarrow 0+}$ do. The eigenvalues of $B_{4}$ can be checked numerically. Allowed regions in parameter space are show in Fig. 7 for $\tan \beta=1$ (left) and $\tan \beta=5$ (right), for several values of $\lambda_{S}$. For this plot we only show the regions for $\xi=2$, as very small region of the parameter space satisfies the perturbativity conditions for $\xi=1^{13}$. This happens because of our choice of imposing the CP2 symmetry, removing the freedom of $\lambda_{3}$. We also set the value of $\lambda_{4}, \lambda_{5}$ to the ones that maximise the allowed parameter space.

The perturbativity constraints on $\lambda_{4,5}$ imply that the mass splitting between the charged scalar and the pseudoscalar can have a maximum allowed value, that is shown in Fig. 8 as a function of the charged scalar mass for $\xi=1,2$.

Note that the mass splitting in the additional doublet affects also EW oblique parameters at loop level. The most stringent constraint comes from the $T$ parameter [86] and usually imposes much more stringent constraints on the maximum splittings $\Delta_{A+}=M_{A}^{2}-M_{H^{+}}^{2}, \Delta_{S_{1,2}+}=M_{S_{1,2}}^{2}-M_{H^{+}}^{2}$ and the mixing angle $\theta$ than the ones obtained by the perturbativity limits on the couplings.

All the plots in this section account for the following necessary global stability conditions:

$$
\begin{aligned}
\lambda_{1,2, S} & >0, \\
\sqrt{\lambda_{1} \lambda_{2}} & >-\lambda_{3}, \\
\sqrt{2 \lambda_{1} \lambda_{S}} & >-\lambda_{11 S}, \\
\sqrt{2 \lambda_{2} \lambda_{S}} & >-\lambda_{22 S}, \\
\sqrt{\lambda_{1} \lambda_{2}} & >\left|\lambda_{5}\right|-\lambda_{3}-\lambda_{4} .
\end{aligned}
$$

\footnotetext{
${ }^{13}$ Varying the value of $\xi$ results in an approximate shrinking of the regions by a $\xi$-dependent factor. The critical point below which the regions shrink to the origin is $\xi=0.9$.
} 


\section{B RGEs for 2-gen 2HDM+S}

2HDM bring in the picture a lot of constraints from flavour physics. Even with our MFV approach, there are two main sources of new flavour physics: First, the presence of a new charged scalar [70], and second the RGE running of the Yukawa couplings [76]. In models with NFC coming from a $Z_{2}$ symmetry, the structure of the couplings is stable under quantum corrections and this point does not apply [87]. Updated constrains for the $Z_{2}$ and aligned model can be found in [71].

The 2-gen model suppresses constraints coming from loop diagrams involving $t, b$ quarks running in the loop and $b, t$ quarks as external particles thanks to it coupling structure. We analyse here RGE runnings to check that they are indeed stable. We can write the RGE equations for a $2 \mathrm{HDM}$, in their most general form, as

$$
\begin{aligned}
16 \pi^{2} \frac{d}{d \ln \mu} \tilde{Y}_{k}^{u}(\mu)= & \left\{N_{\mathrm{c}} \sum_{\ell=h, H} \operatorname{Tr}\left[\tilde{Y}_{k}^{u} \tilde{Y}_{\ell}^{u \dagger}+\tilde{Y}_{\ell}^{d} \tilde{Y}_{k}^{d \dagger}\right] \tilde{Y}_{\ell}^{u}+\frac{1}{2} \sum_{\ell=h, H}\left[\tilde{Y}_{\ell}^{u} \tilde{Y}_{\ell}^{u \dagger}+\tilde{Y}_{\ell}^{d} \tilde{Y}_{\ell}^{d \dagger}\right] \tilde{Y}_{k}^{u}\right. \\
& \left.+\tilde{Y}_{k}^{u} \sum_{\ell=h, H} \tilde{Y}_{\ell}^{u \dagger} \tilde{Y}_{\ell}^{u}-2 \sum_{\ell=h, H}\left[\tilde{Y}_{\ell}^{d} \tilde{Y}_{k}^{d \dagger} \tilde{Y}_{\ell}^{u}\right]-A_{U} \tilde{Y}_{k}^{u}\right\}, \\
16 \pi^{2} \frac{d}{d \ln \mu} \tilde{Y}_{k}^{d}(\mu)= & \left\{N_{\mathrm{c}} \sum_{\ell=h, H} \operatorname{Tr}\left[\tilde{Y}_{k}^{d} \tilde{Y}_{\ell}^{d \dagger}+\tilde{Y}_{\ell}^{u} \tilde{Y}_{k}^{u \dagger}\right] \tilde{Y}_{\ell}^{d}+\frac{1}{2} \sum_{\ell=h, H}\left[\tilde{Y}_{\ell}^{d} \tilde{Y}_{\ell}^{d \dagger}+\tilde{Y}_{\ell}^{u} \tilde{Y}_{\ell}^{u \dagger}\right] \tilde{Y}_{k}^{d}\right. \\
& \left.+\tilde{Y}_{k}^{d} \sum_{\ell=h, H} \tilde{Y}_{\ell}^{d \dagger} \tilde{Y}_{\ell}^{d}-2 \sum_{\ell=h, H}\left[\tilde{Y}_{\ell}^{u} \tilde{Y}_{k}^{u \dagger} \tilde{Y}_{\ell}^{d}\right]-A_{D} \tilde{Y}_{k}^{d}\right\},
\end{aligned}
$$

where $k=h, H$ and

$$
\begin{aligned}
& A_{U}=8 g_{3}^{2}+\frac{9}{4} g_{2}^{2}+\frac{17}{12} g_{1}^{2}, \\
& A_{D}=A_{U}-g_{1}^{2}
\end{aligned}
$$

We can now rewrite them under the assumption that the Yukawa matrices are approximately of the form

$$
\begin{aligned}
Y_{H}^{U} & =V^{\dagger}\left(A P_{12}+a \lambda_{F C}^{U}\right), \\
Y_{H}^{D} & =B P_{12}+b \lambda_{F C}^{D}, \\
Y_{h}^{U} & =V^{\dagger}\left(y_{t} P_{3}+c \lambda_{F C}^{U}\right), \\
Y_{h}^{D} & =y_{b} P_{3}+d \lambda_{F C}^{D},
\end{aligned}
$$

where

$$
\begin{aligned}
& \lambda_{F C}^{U}=V P_{12} V^{\dagger} P_{3}, \\
& \lambda_{F C}^{D}=P_{12} V P_{3} V^{\dagger},
\end{aligned}
$$

and $a, b, c, d$ are zero at some scale and always very small at any relevant scale. Under this assumption, neglecting $a, b, c, d$ terms on the RHS of the RGE equations, together with terms of order $O\left(y_{c}, y_{s}, y_{d}, y_{u}, \frac{y_{b}^{2}}{y_{t}^{2}}\right)$, the set of equations can be solved in sequence:

$$
16 \pi^{2} \frac{d y_{t}}{d \log E}=\left(\frac{9}{2} y_{t}^{2}-A_{U}\right) y_{t}
$$




$$
\begin{aligned}
16 \pi^{2} \frac{d y_{b}}{d \log E} & =\left(\frac{3}{2} y_{t}^{2}-A_{D}\right) y_{b} \\
16 \pi^{2} \frac{d A}{d \log E} & =\left(\frac{15}{2} A^{2}+\frac{9}{2} B^{2}-A_{U}\right) A \\
16 \pi^{2} \frac{d B}{d \log E} & =\left(\frac{15}{2} B^{2}+\frac{9}{2} A^{2}-A_{D}\right) B \\
16 \pi^{2} \frac{d a}{d \log E} & =\left(\frac{1}{2} y_{b}^{2}+\frac{3}{2} B^{2}\right) A \\
16 \pi^{2} \frac{d b}{d \log E} & =\left(\frac{1}{2} y_{t}^{2}+\frac{3}{2} A^{2}\right) B \\
16 \pi^{2} \frac{d c}{d \log E} & =\left(\frac{1}{2} B^{2}+\frac{3}{2} y_{b}^{2}\right) y_{t} \\
16 \pi^{2} \frac{d d}{d \log E} & =\left(\frac{1}{2} A^{2}+\frac{3}{2} y_{t}^{2}\right) y_{b} .
\end{aligned}
$$

The running of gauge coupling is described by

$$
\begin{aligned}
16 \pi^{2} \frac{d g_{i}}{d \log E} & =-C_{i} g_{i}^{3}, \\
C_{3}=\frac{1}{3}\left(11 N_{c}-2 n_{q}\right), \quad C_{2} & =7-\frac{2}{3} n_{q}, \quad C_{1}=-\frac{1}{3}-\frac{10}{9} n_{q},
\end{aligned}
$$

and we fix

$$
\begin{aligned}
\alpha_{3}\left(M_{Z}\right) & =0.118 \\
\alpha_{2}\left(M_{Z}\right) & =0.0332 \\
\alpha_{1}\left(M_{Z}\right) & =0.0101 \\
y_{t}\left(\tilde{m}_{t}\right) & =\frac{\sqrt{2} \tilde{m}_{t}}{v}, \quad \tilde{m}_{t}=166 \mathrm{GeV}, \\
y_{b}\left(m_{b}\right) & =\frac{\sqrt{2} m_{b}}{v}, \quad m_{b}=4.3 \mathrm{GeV} .
\end{aligned}
$$

Note that $\tilde{m}_{t}$ has been chosen as in [76] and

$$
\begin{aligned}
A\left(\tilde{m}_{t}\right) & =0.1 \\
B\left(\tilde{m}_{t}\right) & =0.01, \\
a\left(\tilde{m}_{t}\right) & =b\left(\tilde{m}_{t}\right)=c\left(\tilde{m}_{t}\right)=d\left(\tilde{m}_{t}\right)=0 .
\end{aligned}
$$

The behaviour of the couplings is shown in Fig. 9. The resulting values of the flavour-violating coefficients are approximately ${ }^{14}$

$$
\begin{aligned}
& \left|a\left(m_{b}\right)\right|=1.2 \times 10^{-5} A\left(\tilde{m}_{t}\right), \\
& \left|b\left(m_{b}\right)\right|=1.6 \times 10^{-2} B\left(\tilde{m}_{t}\right),
\end{aligned}
$$

\footnotetext{
${ }^{14}$ Note that if $A$ and $B$ are sufficiently small, equations for $A, B, a, b$ are linear in $A, B$ and equations for $c, d$ are linear in $A^{2}, B^{2}$, thus for any starting value such that $A^{2} \ll y_{t}^{2}, B^{2} \ll y_{b}^{2}$, this is a suitable approximation.
} 


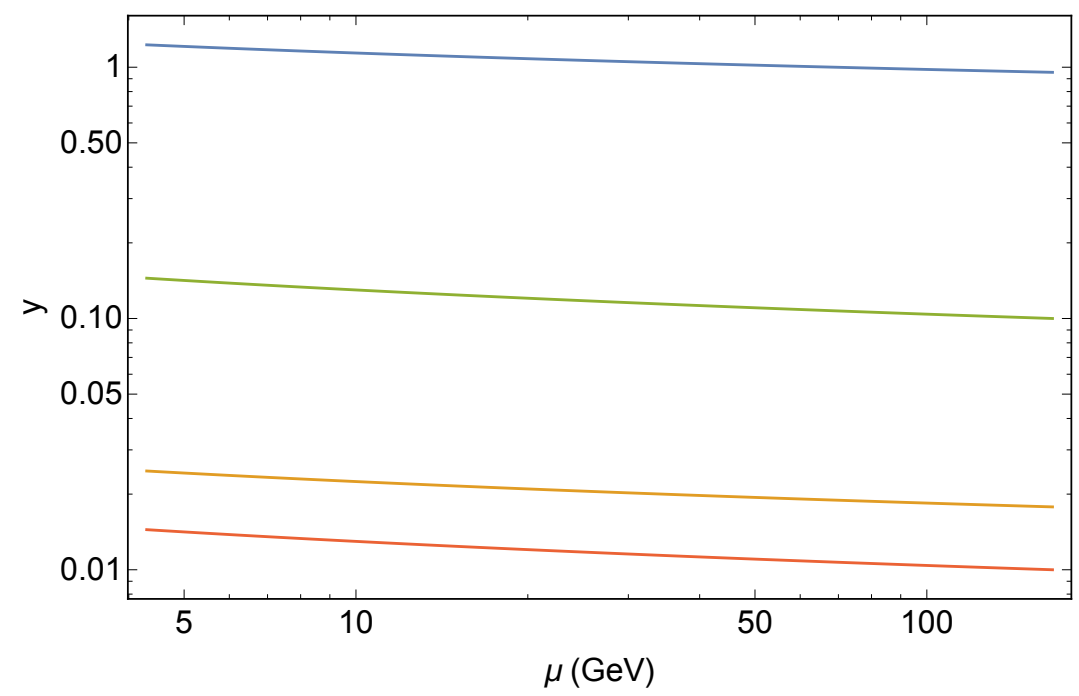

Figure 9: Yukawa couplings as a function of the energy scale $\mu$. From top to bottom: $y_{t}, A, y_{b}, B$. With these initial values the hypothesis $A^{2} \ll y_{t}^{2}$ and $B^{2} \ll y_{b}^{2}$ is valid at all energy scales.

$$
\begin{aligned}
& \left|c\left(m_{b}\right)\right|=1.8 \times 10^{-2} B^{2}\left(\tilde{m}_{t}\right)+5.4 \times 10^{-6}, \\
& \left|d\left(m_{b}\right)\right|=3.5 \times 10^{-4} A^{2}\left(\tilde{m}_{t}\right)+7.4 \times 10^{-4} .
\end{aligned}
$$

Of these, the most dangerous contribution is the coefficient $b\left(m_{b}\right)$, that generates $b \rightarrow s$ FCNC. Using the limit taken from [88], one has

$$
\frac{b\left(m_{b}\right)}{0.01} \times 500 \mathrm{GeV} \sqrt{\frac{\cos ^{2} \theta}{M_{S_{1}}^{2}}+\frac{\sin ^{2} \theta}{M_{S_{2}}^{2}}} \lesssim 3,
$$

which is always satisfied under our assumption $B\left(\tilde{m}_{t}\right) \lesssim y_{b}$.

\section{Near-alignment and the $V_{\text {СКм }}$ pattern}

We now decide to work in a different framework. We can drop the alignment condition we imposed in Sec. 3.1, and instead suppose only an approximate alignment. This means, in the usual 2HDM formalism, that

$$
\left(\begin{array}{c}
\rho_{1} \\
\rho_{2}
\end{array}\right)=R\left(\frac{\pi}{2}-\alpha\right)\left(\begin{array}{c}
h \\
H
\end{array}\right),
$$

where $h, H$ are the two mass eigenstates, and $h$ is the $125 \mathrm{GeV}$ SM Higgs boson. In this case the SM Higgs $h_{\mathrm{SM}}$ will not be a mass eigenstate, like when we have perfect alignment, but rather a mixture, as it is related to the Higgs basis by a rotation

$$
\left(\begin{array}{c}
h_{\mathrm{SM}} \\
H_{\perp}
\end{array}\right)=R(\beta)\left(\begin{array}{c}
\rho_{1} \\
\rho_{2}
\end{array}\right)=R(\beta) R\left(\frac{\pi}{2}-\alpha\right)\left(\begin{array}{c}
h \\
H
\end{array}\right)=R\left(\beta-\alpha+\frac{\pi}{2}\right)\left(\begin{array}{c}
h \\
H
\end{array}\right),
$$

where $H_{\perp}$ is the linear combination orthogonal to the SM one. We can then rewrite Eq. (3.9), (3.10) as

$$
\Phi_{h}=\cos \beta \Phi_{1}+\sin \beta \Phi_{2}=\left(\begin{array}{c}
G^{+} \\
\frac{1}{\sqrt{2}}\left(v+\sin (\alpha-\beta) h_{\mathrm{SM}}-\cos (\alpha-\beta) H_{\perp}+G^{0}\right)
\end{array}\right),
$$




\begin{tabular}{|c|cc|}
\hline Particle & $U(1)$ & $Z_{4}$ \\
\hline$\Phi_{h}^{\prime}$ & 0 & 0 \\
$\Phi_{H}^{\prime}$ & 1 & 1 \\
$S$ & 1 & 1 \\
$Q_{L}^{3}$ & $m+3$ & $m-1$ \\
$Q_{L}^{1,2}$ & $m-1$ & $m$ \\
$u_{R}^{3}$ & $m+3$ & $m-1$ \\
$u_{R}^{1,2}$ & $m+1$ & $m+1$ \\
$d_{R}^{3}$ & $m+3$ & $m-1$ \\
$d_{R}^{1,2}$ & $m-1$ & $m-1$ \\
$\chi_{L}$ & 1 & 1 \\
$\chi_{R}$ & 0 & 0 \\
\hline
\end{tabular}

Table 3: Example of choice of charges for the global symmetry. Here $m \in \mathbb{Z}$.

$$
\Phi_{H}=-\sin \beta \Phi_{1}+\cos \beta \Phi_{2}=\left(\begin{array}{c}
H^{+} \\
\frac{1}{\sqrt{2}}\left(\cos (\alpha-\beta) h_{\mathrm{SM}}+\sin (\alpha-\beta) H_{\perp}+A\right)
\end{array}\right) .
$$

The near-alignment will be defined as

$$
0 \neq \cos (\beta-\alpha) \ll 1 \rightarrow \alpha-\beta-\pi / 2=\epsilon \ll 1,
$$

and we can perform a rotation to go to the basis where the doublets contain the neutral scalar mass eigenstates

$$
\begin{aligned}
& \Phi_{h}^{\prime}=\cos \epsilon \Phi_{h}-\sin \epsilon \Phi_{H}=\left(\begin{array}{c}
\cos \epsilon G^{+}-\sin \epsilon H^{+} \\
\frac{1}{\sqrt{2}}\left(v \cos \epsilon+h_{\mathrm{SM}}+\cos \epsilon G^{0}-\sin \epsilon A\right)
\end{array}\right), \\
& \Phi_{H}^{\prime}=\sin \epsilon \Phi_{h}+\cos \epsilon \Phi_{H}=\left(\begin{array}{c}
\cos \epsilon H^{+}+\sin \epsilon G^{+} \\
\frac{1}{\sqrt{2}}\left(v \sin \epsilon+H_{\perp}+\cos \epsilon A+\sin \epsilon G^{0}\right)
\end{array}\right),
\end{aligned}
$$

where $\epsilon$ is such that ${ }^{15}$

$$
1 \mathrm{GeV} \lesssim v \sin \epsilon \lesssim 100 \mathrm{GeV} \rightarrow 2.5 \lesssim \tan (\beta-\alpha) \lesssim 250
$$

We can then assume the Lagrangian has either an additional global $U(1)$ or $Z_{4}$ symmetry, with the charges listed in Tab. 3 (the real scalar needs to be promoted to a complex scalar to give it a $U(1)$ charge). With such symmetries SM fermions are forced to couple only to one of the two doublets of Eq. (C.6), (C.7). We can use these symmetries to discard some terms in the scalar potential, even though we may want to retain soft breaking terms. With the $Z_{4}$ symmetry, we can see that this model is equivalent to up-type BGL models [89]. Thus FCNC are absent at tree level in the up sector, and are only present in the down sector, suppressed by CKM matrix entries. The patterns of the $U_{u \mid L, R}$ matrices that diagonalise the Yukawa matrices are, in the case of the $Z_{4}$ symmetry, are

$$
U_{u \mid L, R}=\left(\begin{array}{ccc}
\times & \times & 0 \\
\times & \times & 0 \\
0 & 0 & 1
\end{array}\right)
$$

\footnotetext{
${ }^{15}$ The lower limit comes from the requirement of perturbativity for the charm quark Yukawa coupling for the new doublet.
} 
while the patterns of the Yukawa matrices are

$$
Y_{1}^{U}=\left(\begin{array}{ccc}
0 & 0 & 0 \\
0 & 0 & 0 \\
0 & 0 & \times
\end{array}\right), Y_{2}^{U}=\left(\begin{array}{ccc}
\times & \times & 0 \\
\times & \times & 0 \\
0 & 0 & 0
\end{array}\right), Y_{1}^{D}=\left(\begin{array}{ccc}
0 & 0 & 0 \\
0 & 0 & 0 \\
\times & \times & \times
\end{array}\right), Y_{2}^{D}=\left(\begin{array}{ccc}
\times & \times & \times \\
\times & \times & \times \\
0 & 0 & 0
\end{array}\right)
$$

In this way, we can choose not only $y_{t}$, but also $y_{c}$ to be of order one, and thus the smallness of the lighter quark masses could be explained not by a tiny value of their Yukawa coupling, but rather by the smallness of $v_{2}$. In this context, the coupling of the charm quark to the $125 \mathrm{GeV}$ Higgs boson would result in being much smaller than the SM, but this is compatible with current experimental results [90].

Moreover, in [91] there is a claim of the presence of fine-tuning in the CKM matrix, given the smallness of $\left|V_{13}\right|^{2}+\left|V_{23}\right|^{2}$. This framework complied with one of the possible explanations of this alignment present in the CKM matrix proposed in [91] - however the magnitude of FCNC both at tree and loop level of this model should be analysed and may provide additional constrains on the allowed values of $\epsilon$.

The BGL model induces such FCNC at tree level with couplings for the second doublet given by $^{16}$ the off-diagonal elements of

$$
\frac{N_{d}+N_{d}^{\dagger}}{2 v}
$$

which are

$$
\frac{m_{b}}{v \sin 2 \epsilon}\left(\begin{array}{ccc}
0 & 0 & V_{31}^{*} \\
0 & 0 & V_{32}^{*} \\
V_{31} & V_{32} & 0
\end{array}\right)
$$

where $N_{d}$ is the matrix defined in [31]:

$$
N_{d}=\frac{1}{\sqrt{2}} U_{d L}^{\dagger}\left(v_{2} Y_{1}^{D}-v_{1} Y_{2}^{D}\right) U_{d R}
$$

Using the limits from [88] we get that ${ }^{17}$

$$
\sin 2 \epsilon>0.56 \times 500 \mathrm{GeV} \sqrt{\frac{\cos ^{2} \theta}{M_{S_{1}}^{2}}+\frac{\sin ^{2} \theta}{M_{S_{2}}^{2}}} .
$$

For $M_{S_{1}}=500 \mathrm{GeV}, \theta=\pi / 4$ and $M_{S_{2}} \gg M_{S_{1}}$, one has

$$
\sin \epsilon>0.2, \quad \cos \epsilon<0.98, \quad \cot \epsilon=\tan (\beta-\alpha)<4.8 .
$$

\footnotetext{
${ }^{16}$ We neglect $\mathrm{CP}$ violation also here.

${ }^{17}$ Note that here we are considering the most favourable scenario, where $h_{\mathrm{SM}}$ is a mass eigenstate, and $H_{\perp}$ is mixing with the singlet, or, in other words, that $S$, written as linear combination of mass eigenstates, does not contain the $125 \mathrm{GeV}$ Higgs boson. In general, one will need to consider a full $3 \times 3$ mixing for the 3 scalars.
} 


\section{References}

[1] G. Busoni, A. De Simone, E. Morgante, and A. Riotto, "On the Validity of the Effective Field Theory for Dark Matter Searches at the LHC," Phys. Lett. B728 (2014) 412-421, arXiv:1307.2253 [hep-ph].

[2] G. Busoni, A. De Simone, J. Gramling, E. Morgante, and A. Riotto, "On the Validity of the Effective Field Theory for Dark Matter Searches at the LHC, Part II: Complete Analysis for the $s$-channel," JCAP 1406 (2014) 060, arXiv:1402.1275 [hep-ph].

[3] G. Busoni, A. De Simone, T. Jacques, E. Morgante, and A. Riotto, "On the Validity of the Effective Field Theory for Dark Matter Searches at the LHC Part III: Analysis for the $t$-channel," JCAP 1409 (2014) 022, arXiv:1405.3101 [hep-ph].

[4] O. Buchmueller, M. J. Dolan, and C. McCabe, "Beyond Effective Field Theory for Dark Matter Searches at the LHC," JHEP 01 (2014) 025, arXiv:1308.6799 [hep-ph].

[5] J. Abdallah et al., "Simplified Models for Dark Matter and Missing Energy Searches at the LHC," arXiv: 1409.2893 [hep-ph].

[6] I. M. Shoemaker and L. Vecchi, "Unitarity and Monojet Bounds on Models for DAMA, CoGeNT, and CRESST-II," Phys. Rev. D86 (2012) 015023, arXiv:1112.5457 [hep-ph].

[7] J. Abdallah et al., "Simplified Models for Dark Matter Searches at the LHC," Phys. Dark Univ. 9-10 (2015) 8-23, arXiv:1506.03116 [hep-ph].

[8] D. Abercrombie et al., "Dark Matter Benchmark Models for Early LHC Run-2 Searches: Report of the ATLAS/CMS Dark Matter Forum," arXiv:1507.00966 [hep-ex].

[9] G. Busoni et al., "Recommendations on presenting LHC searches for missing transverse energy signals using simplified s-channel models of dark matter," arXiv:1603.04156 [hep-ex].

[10] T. Jacques, A. Katz, E. Morgante, D. Racco, M. Rameez, and A. Riotto, "Complementarity of DM Searches in a Consistent Simplified Model: the Case of Z'," arXiv:1605.06513 [hep-ph].

[11] M. R. Buckley, D. Feld, and D. Goncalves, "Scalar Simplified Models for Dark Matter," Phys. Rev. D91 (2015) 015017, arXiv:1410.6497 [hep-ph].

[12] P. Harris, V. V. Khoze, M. Spannowsky, and C. Williams, "Constraining Dark Sectors at Colliders: Beyond the Effective Theory Approach," Phys. Rev. D91 (2015) 055009, arXiv:1411.0535 [hep-ph].

[13] N. F. Bell, Y. Cai, J. B. Dent, R. K. Leane, and T. J. Weiler, "Dark matter at the LHC: Effective field theories and gauge invariance," Phys. Rev. D92 no. 5, (2015) 053008, arXiv:1503.07874 [hep-ph].

[14] N. F. Bell, Y. Cai, and R. K. Leane, "Mono-W Dark Matter Signals at the LHC: Simplified Model Analysis," JCAP 1601 no. 01, (2016) 051, arXiv:1512.00476 [hep-ph].

[15] U. Haisch, F. Kahlhoefer, and T. M. P. Tait, "On Mono-W Signatures in Spin-1 Simplified Models," Phys. Lett. B760 (2016) 207-213, arXiv:1603.01267 [hep-ph].

[16] C. Englert, M. McCullough, and M. Spannowsky, "S-Channel Dark Matter Simplified Models and Unitarity," arXiv:1604.07975 [hep-ph].

[17] F. Kahlhoefer, K. Schmidt-Hoberg, T. Schwetz, and S. Vogl, "Implications of unitarity and gauge invariance for simplified dark matter models," JHEP 02 (2016) 016, arXiv:1510.02110 [hep-ph].

[18] N. F. Bell, Y. Cai, and R. K. Leane, "Dark Forces in the Sky: Signals from Z' and the Dark Higgs," JCAP 1608 no. 08, (2016) 001, arXiv:1605.09382 [hep-ph].

[19] P. Ko, A. Natale, M. Park, and H. Yokoya, "Simplified DM models with the full SM gauge symmetry : the case of $t$-channel colored scalar mediators," arXiv:1605.07058 [hep-ph]. 
[20] M. Duerr, F. Kahlhoefer, K. Schmidt-Hoberg, T. Schwetz, and S. Vogl, "How to save the WIMP: global analysis of a dark matter model with two s-channel mediators," JHEP 09 (2016) 042, arXiv:1606.07609 [hep-ph].

[21] N. F. Bell, Y. Cai, and R. K. Leane, "Impact of Mass Generation for Simplified Dark Matter Models," arXiv:1610.03063 [hep-ph].

[22] V. V. Khoze, G. Ro, and M. Spannowsky, "Spectroscopy of scalar mediators to dark matter at the LHC and at 100 TeV," Phys. Rev. D92 no. 7, (2015) 075006, arXiv:1505.03019 [hep-ph].

[23] S. Baek, P. Ko, M. Park, W.-I. Park, and C. Yu, "Beyond the Dark matter effective field theory and a simplified model approach at colliders," Phys. Lett. B756 (2016) 289-294, arXiv:1506.06556 [hep-ph].

[24] M. Bauer et al., "Towards the next generation of simplified Dark Matter models," arXiv:1607.06680 [hep-ex].

[25] T. Robens and T. Stefaniak, "LHC Benchmark Scenarios for the Real Higgs Singlet Extension of the Standard Model," Eur. Phys. J. C76 no. 5, (2016) 268, arXiv:1601.07880 [hep-ph].

[26] Z.-W. Wang, T. G. Steele, T. Hanif, and R. B. Mann, "Conformal Complex Singlet Extension of the Standard Model: Scenario for Dark Matter and a Second Higgs Boson," JHEP 08 (2016) 065, arXiv: 1510.04321 [hep-ph].

[27] D. Lpez-Val and T. Robens, "r and the W-boson mass in the singlet extension of the standard model," Phys. Rev. D90 (2014) 114018, arXiv:1406.1043 [hep-ph].

[28] R. Costa, M. Mhlleitner, M. O. P. Sampaio, and R. Santos, "Singlet Extensions of the Standard Model at LHC Run 2: Benchmarks and Comparison with the NMSSM," JHEP 06 (2016) 034, arXiv:1512.05355 [hep-ph].

[29] G. Dupuis, "Collider Constraints and Prospects of a Scalar Singlet Extension to Higgs Portal Dark Matter," JHEP 07 (2016) 008, arXiv:1604.04552 [hep-ph].

[30] C. Balazs, A. Fowlie, A. Mazumdar, and G. White, "Gravitational waves at aLIGO and vacuum stability with a scalar singlet extension of the Standard Model," arXiv:1611.01617 [hep-ph].

[31] G. C. Branco, P. M. Ferreira, L. Lavoura, M. N. Rebelo, M. Sher, and J. P. Silva, "Theory and phenomenology of two-Higgs-doublet models," Phys. Rept. 516 (2012) 1-102, arXiv:1106.0034 [hep-ph].

[32] U. Amaldi, W. de Boer, and H. Furstenau, "Comparison of grand unified theories with electroweak and strong coupling constants measured at LEP," Phys. Lett. B260 (1991) 447-455.

[33] M. Carena, M. Quiros, and C. E. M. Wagner, "Effective potential methods and the Higgs mass spectrum in the MSSM," Nucl. Phys. B461 (1996) 407-436, arXiv:hep-ph/9508343 [hep-ph].

[34] G. Bhattacharyya and D. Das, "Scalar sector of two-Higgs-doublet models: A minireview," Pramana 87 no. 3, (2016) 40, arXiv:1507.06424 [hep-ph].

[35] P. Fayet, "Supersymmetry and Weak, Electromagnetic and Strong Interactions," Phys. Lett. B64 (1976) 159.

[36] J. F. Gunion and H. E. Haber, "Higgs Bosons in Supersymmetric Models. 1.," Nucl. Phys. B272 (1986) 1. [Erratum: Nucl. Phys.B402,567(1993)].

[37] S. F. King, M. Mhlleitner, R. Nevzorov, and K. Walz, "Natural NMSSM Higgs Bosons," Nucl. Phys. B870 (2013) 323-352, arXiv:1211.5074 [hep-ph].

[38] C.-Y. Chen, M. Freid, and M. Sher, "Next-to-minimal two Higgs doublet model," Phys. Rev. D89 no. 7, (2014) 075009, arXiv:1312.3949 [hep-ph]. 
[39] S. Kanemura, M. Kikuchi, and K. Yagyu, "Radiative corrections to the Higgs boson couplings in the model with an additional real singlet scalar field," Nucl. Phys. B907 (2016) 286-322, arXiv:1511.06211 [hep-ph].

[40] S. von Buddenbrock, N. Chakrabarty, A. S. Cornell, D. Kar, M. Kumar, T. Mandal, B. Mellado, B. Mukhopadhyaya, R. G. Reed, and X. Ruan, "Phenomenological signatures of additional scalar bosons at the LHC," Eur. Phys. J. C76 no. 10, (2016) 580, arXiv:1606.01674 [hep-ph].

[41] P. Ko and J. Li, "Interference effects of two scalar boson propagators on the LHC search for the singlet fermion DM," arXiv:1610.03997 [hep-ph].

[42] J. Kumar and D. Marfatia, "Matrix element analyses of dark matter scattering and annihilation," Phys. Rev. D88 no. 1, (2013) 014035, arXiv:1305.1611 [hep-ph].

[43] S. Ipek, D. McKeen, and A. E. Nelson, "A Renormalizable Model for the Galactic Center Gamma Ray Excess from Dark Matter Annihilation," Phys. Rev. D90 no. 5, (2014) 055021, arXiv:1404.3716 [hep-ph].

[44] A. Berlin, S. Gori, T. Lin, and L.-T. Wang, "Pseudoscalar Portal Dark Matter," Phys. Rev. D92 (2015) 015005, arXiv:1502.06000 [hep-ph].

[45] D. Goncalves, P. A. N. Machado, and J. M. No, "Simplified Models for Dark Matter Face their Consistent Completions," arXiv:1611.04593 [hep-ph].

[46] J. M. No, "Looking through the pseudoscalar portal into dark matter: Novel mono-Higgs and mono-Z signatures at the LHC," Phys. Rev. D93 no. 3, (2016) 031701, arXiv:1509.01110 [hep-ph].

[47] M. Bauer, U. Haisch, and F. Kahlhoefer, "Simplified dark matter models with two Higgs doublets: I. Pseudoscalar mediators," arXiv:1701.07427 [hep-ph].

[48] U. Haisch, P. Pani, and G. Polesello, "Determining the CP nature of spin-0 mediators in associated production of dark matter and $t \bar{t}$ pairs," arXiv:1611.09841 [hep-ph].

[49] ATLAS Collaboration, G. Aad et al., "Search for invisible decays of the Higgs boson produced in association with a hadronically decaying vector boson in $p p$ collisions at $\sqrt{s}=8$ TeV with the ATLAS detector," Eur. Phys. J. C75 no. 7, (2015) 337, arXiv:1504.04324 [hep-ex].

[50] CMS Collaboration, S. Chatrchyan et al., "Search for invisible decays of Higgs bosons in the vector boson fusion and associated ZH production modes," Eur. Phys. J. C74 (2014) 2980, arXiv:1404.1344 [hep-ex].

[51] "Measurements of the Higgs boson production and decay rates and constraints on its couplings from a combined ATLAS and CMS analysis of the LHC pp collision data at $\sqrt{s}=7$ and 8 TeV," Tech. Rep. ATLAS-CONF-2015-044, CERN, Geneva, Sep, 2015. http://cds.cern.ch/record/2052552.

[52] CMS Collaboration, C. Collaboration, "Search for Dark Matter produced in association with bottom quarks,".

[53] CMS Collaboration, C. Collaboration, "Search for dark matter in final states with an energetic jet, or a hadronically decaying $\mathrm{W}$ or Z boson using $12.9 \mathrm{fb}^{-1}$ of data at $\sqrt{s}=13 \mathrm{TeV}, "$

[54] CMS Collaboration, C. Collaboration, "Search for dark matter in association with a top quark pair at $\operatorname{sqrt}(\mathrm{s})=13 \mathrm{TeV}, "$.

[55] ATLAS Collaboration Collaboration, "Search for Dark Matter production associated with bottom quarks with $13.3 \mathrm{fb} 1$ of pp collisions at $\mathrm{s}=13 \mathrm{TeV}$ with the ATLAS detector at the LHC," Tech. Rep. ATLAS-CONF-2016-086, CERN, Geneva, Aug, 2016. http://cds.cern.ch/record/2206279.

[56] ATLAS Collaboration Collaboration, "Search for the Supersymmetric Partner of the Top Quark in 
the Jets+Emiss Final State at sqrt(s) $=13 \mathrm{TeV}$," Tech. Rep. ATLAS-CONF-2016-077, CERN, Geneva, Aug, 2016. http://cds.cern.ch/record/2206250.

[57] J. F. Gunion and H. E. Haber, "The CP conserving two Higgs doublet model: The Approach to the decoupling limit," Phys. Rev. D67 (2003) 075019, arXiv:hep-ph/0207010 [hep-ph].

[58] M. Carena, I. Low, N. R. Shah, and C. E. M. Wagner, "Impersonating the Standard Model Higgs Boson: Alignment without Decoupling," JHEP 04 (2014) 015, arXiv:1310.2248 [hep-ph].

[59] P. S. Bhupal Dev and A. Pilaftsis, "Maximally Symmetric Two Higgs Doublet Model with Natural Standard Model Alignment," JHEP 12 (2014) 024, arXiv:1408.3405 [hep-ph]. [Erratum: JHEP11,147(2015)].

[60] P. S. B. Dev and A. Pilaftsis, "Natural Standard Model Alignment in the Two Higgs Doublet Model," J. Phys. Conf. Ser. 631 no. 1, (2015) 012030, arXiv:1503.09140 [hep-ph].

[61] A. Pilaftsis, "Symmetries for standard model alignment in multi-Higgs doublet models," Phys. Rev. D93 no. 7, (2016) 075012, arXiv:1602.02017 [hep-ph].

[62] P. Draper, H. E. Haber, and J. T. Ruderman, "Partially Natural Two Higgs Doublet Models," JHEP 06 (2016) 124, arXiv:1605.03237 [hep-ph].

[63] G. D'Ambrosio, G. F. Giudice, G. Isidori, and A. Strumia, "Minimal flavor violation: An Effective field theory approach," Nucl. Phys. B645 (2002) 155-187, arXiv:hep-ph/0207036 [hep-ph].

[64] A. J. Buras, M. V. Carlucci, S. Gori, and G. Isidori, "Higgs-mediated FCNCs: Natural Flavour Conservation vs. Minimal Flavour Violation," JHEP 10 (2010) 009, arXiv:1005.5310 [hep-ph].

[65] A. Pich and P. Tuzon, "Yukawa Alignment in the Two-Higgs-Doublet Model," Phys. Rev. D80 (2009) 091702, arXiv:0908.1554 [hep-ph].

[66] P. Tuzon and A. Pich, "The Aligned two-Higgs Doublet model," Acta Phys. Polon. Supp. 3 (2010) 215-220, arXiv:1001.0293 [hep-ph].

[67] A. Pich, "Flavour constraints on multi-Higgs-doublet models: Yukawa alignment," Nucl. Phys. Proc. Suppl. 209 (2010) 182-187, arXiv:1010.5217 [hep-ph].

[68] C. B. Braeuninger, A. Ibarra, and C. Simonetto, "Radiatively induced flavour violation in the general two-Higgs doublet model with Yukawa alignment," Phys. Lett. B692 (2010) 189-195, arXiv: 1005.5706 [hep-ph].

[69] F. J. Botella, G. C. Branco, A. M. Coutinho, M. N. Rebelo, and J. I. Silva-Marcos, "Natural Quasi-Alignment with two Higgs Doublets and RGE Stability," Eur. Phys. J. C75 (2015) 286, arXiv: 1501.07435 [hep-ph].

[70] M. Jung, A. Pich, and P. Tuzon, "Charged-Higgs phenomenology in the Aligned two-Higgs-doublet model," JHEP 11 (2010) 003, arXiv:1006.0470 [hep-ph].

[71] T. Enomoto and R. Watanabe, "Flavor constraints on the Two Higgs Doublet Models of $Z_{2}$ symmetric and aligned types," JHEP 05 (2016) 002, arXiv:1511.05066 [hep-ph].

[72] J. L. Evans, B. Feldstein, W. Klemm, H. Murayama, and T. T. Yanagida, "Hermitian Flavor Violation," Phys. Lett. B703 (2011) 599-605, arXiv:1106.1734 [hep-ph].

[73] W. Altmannshofer, J. Eby, S. Gori, M. Lotito, M. Martone, and D. Tuckler, "Collider Signatures of Flavorful Higgs Bosons," Submitted to: Phys. Rev. D (2016), arXiv:1610.02398 [hep-ph].

[74] M. Arroyo, J. L. Diaz-Cruz, E. Diaz, and J. A. Orduz-Ducuara, "Flavor Violating Higgs signals in the Texturized Two-Higgs Doublet Model (2HDM-Tx)," arXiv:1306.2343 [hep-ph]. 
[75] F. Bishara, J. Brod, P. Uttayarat, and J. Zupan, "Nonstandard Yukawa Couplings and Higgs Portal Dark Matter," JHEP 01 (2016) 010, arXiv:1504.04022 [hep-ph].

[76] G. Cvetic, C. S. Kim, and S. S. Hwang, "Higgs mediated flavor changing neutral currents in the general framework with two Higgs doublets: An RGE analysis," Phys. Rev. D58 (1998) 116003, arXiv:hep-ph/9806282 [hep-ph].

[77] B. R. Desai and A. R. Vaucher, "Solutions to the renormalization group equations for Yukawa matrices as an answer to the quark and lepton mass problem," arXiv:hep-ph/0309102 [hep-ph].

[78] Y. G. Kim, K. Y. Lee, and S. Shin, "Singlet fermionic dark matter," JHEP 05 (2008) 100, arXiv:0803.2932 [hep-ph].

[79] S. Baek, P. Ko, and W.-I. Park, "Search for the Higgs portal to a singlet fermionic dark matter at the LHC," JHEP 02 (2012) 047, arXiv:1112.1847 [hep-ph].

[80] L. Lopez-Honorez, T. Schwetz, and J. Zupan, "Higgs portal, fermionic dark matter, and a Standard Model like Higgs at $125 \mathrm{GeV}, "$ Phys. Lett. B716 (2012) 179-185, arXiv:1203.2064 [hep-ph].

[81] M. Cirelli, E. Del Nobile, and P. Panci, "Tools for model-independent bounds in direct dark matter searches," JCAP 1310 (2013) 019, arXiv:1307.5955 [hep-ph].

[82] LUX Collaboration, D. S. Akerib et al., "First results from the LUX dark matter experiment at the Sanford Underground Research Facility," Phys. Rev. Lett. 112 (2014) 091303, arXiv:1310.8214 [astro-ph. CO].

[83] J. Ellis, "TikZ-Feynman: Feynman diagrams with TikZ," arXiv:1601.05437 [hep-ph].

[84] N. Bell, G. Busoni, A. Kobakhidze, D. M. Long, and M. A. Schmidt, "Unitarisation of EFT Amplitudes for Dark Matter Searches at the LHC," JHEP 08 (2016) 125, arXiv:1606.02722 [hep-ph].

[85] S. Kanemura and K. Yagyu, "Unitarity bound in the most general two Higgs doublet model," Phys. Lett. B751 (2015) 289-296, arXiv:1509.06060 [hep-ph].

[86] D. Lpez-Val, T. Plehn, and M. Rauch, "Measuring extended Higgs sectors as a consistent free couplings model," JHEP 10 (2013) 134, arXiv:1308.1979 [hep-ph].

[87] P. M. Ferreira, L. Lavoura, and J. P. Silva, "Renormalization-group constraints on Yukawa alignment in multi-Higgs-doublet models," Phys. Lett. B688 (2010) 341-344, arXiv:1001.2561 [hep-ph].

[88] A. Crivellin, A. Kokulu, and C. Greub, "Flavor-phenomenology of two-Higgs-doublet models with generic Yukawa structure," Phys. Rev. D87 no. 9, (2013) 094031, arXiv:1303.5877 [hep-ph].

[89] G. C. Branco, W. Grimus, and L. Lavoura, "Relating the scalar flavor changing neutral couplings to the CKM matrix," Phys. Lett. B380 (1996) 119-126, arXiv:hep-ph/9601383 [hep-ph].

[90] F. Bishara, U. Haisch, P. F. Monni, and E. Re, "Constraining Light-Quark Yukawa Couplings from Higgs Distributions," arXiv:1606.09253 [hep-ph].

[91] F. J. Botella, G. C. Branco, M. N. Rebelo, and J. I. Silva-Marcos, "What if the Masses of the First Two Quark Families are not Generated by the Standard Higgs?," arXiv:1602.08011 [hep-ph]. 